\title{
Determination of pesticide residues in honey: a preliminary study from two of Africa's largest honey producers
}

\author{
Janet Irungu*, Suresh Raina and Baldwyn Torto
}

\begin{abstract}
Background: The presence of pollutants in honey can influence honey bee colony performance and devalue its use for human consumption. Using liquid chromatography tandem mass spectrometry (LC-MS/MS), various clean-up methods were evaluated for efficient determination of multiclass pesticide contaminants in honey. The selected clean-up method was optimized and validated and then applied to perform a preliminary study of commercial honey samples from Africa.

Results: The most efficient method was primary-secondary amine (PSA) sorbent which was significantly different from the others $(P<0.05$; average recovery $\sim 94 \%)$ and was applied to analyze 96 pesticide residues in 28 retail honey samples from Kenya and Ethiopia. From our preliminary data, a total of 17 pesticide residues were detected at $\sim 10$-fold below maximum residue limit (MRL) established for food products except for malathion which was detected at almost 2-fold above its acceptable MRL.

Conclusions: A highly efficient approach for determining pesticide residues in honey with good recoveries was developed. All residue contaminants were detected at levels well below their acceptable MRLs except malathion suggesting that the retail honey analyzed is safe for human consumption. Although PSA clean-up method was selected as the most efficient for cleaning honey samples, omitting the clean-up step was the most economical approach with potential applicability in the food industry.
\end{abstract}

Keywords: Pesticide residues, Honey bees, Liquid chromatography-tandem mass spectrometry (LC-MS/MS), Honey, Method development

\section{Background}

The recent sudden decline of honey bee colonies is of global concern not only because of pollination services they provide in food production process, but also due to honey production among other benefits. While there are multiple variables, including poor nutrition, pests, diseases, and loss of natural bee habitat, negatively affecting bee health, it is becoming increasingly clear that the widespread use of pesticides on agricultural crops is a major factor (Vanengelsdorp and Meixner 2010; Gill et al. 2012; Brodschneider and Crailsheim 2010). As such, to preserve honey bee health which is inextricably integrated with human health and to preserve the quality of bee by-products

\footnotetext{
* Correspondence: jirungu@icipe.org

African Reference Laboratory for Bee Health, International Centre of Insect Physiology and Ecology (icipe), P.O. Box 30772-00100, Nairobi, Kenya
}

especially honey, requires regular monitoring using rigorous analytical methods to confirm product quality (Muli et al. 2014; Kujawski and Namiesnik 2008).

Honey is composed of over 300 compounds, mostly carbohydrates $(>75 \%)$ and water $(\sim 18 \%)$, with minor components comprising of proteins, amino acids, vitamins, antioxidants, minerals, essential oils, sterols, pigments, phospholipids, and organic acids (Bogdanov et al. 2008; Kujawski and Namiesnik 2008). Whereas these diverse ranges of compounds make it a nutrient rich food commodity, they also make it a highly complex analytical matrix especially when analysing the presence of trace compounds such as toxins, pesticide residues and other environmental pollutants (Kujawski and Namiesnik 2008). The presence of pesticide residues and other contaminants in honey can have adverse health effects on bees and humans, decrease the quality of honey 
and devalue its beneficial properties (Bogdanov et al. 2008). Typically, pesticide residues in honey occurs when bees in search for food, visit crops that have been treated with various agro-chemicals and/or when beekeepers use chemicals to control bee pests or diseases (Bogdanov 2006). So far, several researchers have reported various residues of pesticides in honey at varying concentrations (De Pinho, et al. 2010; Irani 2009; Barganska et al. 2013; Blasco et al. 2011; Garcia-Chao et al. 2010; Herrera et al. 2005; Rissato et al. 2007; Weist et al. 2011; Fontana et al. 2010; Kujawski and Namiesnik 2011; Wang et al. 2010; Campillo et al. 2006; Choudhary and Sharma 2008; Martel et al. 2007; Erdog`rul 2007; Blasco et al. 2003) confirming the need to constantly monitor the presence of pesticide residues in honey to assess any potential health risk and to ensure that its quality, whether as food or as a therapeutic, is not compromised. However, to date, only few studies have been carried out to monitor pesticide residues in honey produced from Africa (Eissa et al. 2014). A recent study conducted in Kenya in 2010 detected four pesticides from beeswax and bee bread at very low concentrations (Muli et al. 2014). However, the cumulative levels and presence of pesticides in hive products over time can pose health problems for both honeybees and humans. Therefore there is the need to develop highly sensitive and selective analytical techniques that have the ability to analyze multiple pesticides simultaneously in hive products.

Since honey is a complex analytical matrix, it is often necessary to clean-up the sample prior to instrumental analysis (Kujawski and Namiesnik 2008). This facilitates removal of matrix co-extractives that could result in enhancement or suppression of the signal of the targeted analytes during analysis (Ferrer et al. 2011; Kittlaus et al. 2011; Kruve et al. 2008). Conversely, this clean-up step is usually the most expensive, time consuming and laborious sample preparation step with the highest probability of introducing errors on recovery and method repeatability. Conventional extraction/clean-up methods such as liquid-liquid (LLE) or solid-phase extractions (SPE), require large volumes of organic solvents and usually target pesticides from a single chemical class (Fontana et al. 2010; Fernández and Simal 1991; Wang et al. 2010; Martel et al. 2007). Recently, extensive research has been geared towards finding more economical and environmental friendly methods that can yield good recoveries for a diverse range of pesticides. For instance, a recent study compared four different methods for extracting 12 organophosphates and carbamates from honey and concluded that the choice of the method depends on the targeted analytes (Blasco, et al. 2011). In another example (Kujawski et al. 2014), two methods; solid supported liquid-liquid extraction(SLE) and a modified Quick, Easy, Cheap, Effective and Safe (QuEChERS) method for multiresidue analysis were compared using extraction efficiencies for determination of 30 LCamenable pesticides in honey at their MRLs. These authors concluded that in terms of recovery (ranged from 34 to $96 \%)$ the methods had no significant difference but in terms of costs and time, the modified QuEChERS was better (Kujawski et al. 2014). In this study, an ultra-high performance liquid chromatography coupled to tandem mass spectrometry (LC-MS/MS) was employed to analyze multiclass chemical contaminants in African honey at parts per billion (ppb) levels. Four different clean-up methods including PSA plus graphitized carbon (GCB), PSA plus C18, PSA alone, and a no clean-up approach were investigated using 96 LC-amenable pesticides to determine their applicability in a multiclass residue analysis in honey by comparing their recoveries. The method was validated and applied to conduct a preliminary study of pesticide residues in commercial honey samples obtained from Kenya and Ethiopia which are among the major producers of honey in Africa. Previous data on honey production in Africa indicates that Ethiopia is the largest producer with an estimate of 41,233 tons of honey followed by Tanzania at 28,678 tons and Kenya at 25,000 tons in 2004- 2006 (FAOSTAT). To the best of our knowledge, this is the first in-depth multiclass pesticide residue analysis of commercial honey from Africa. These results provide some insights in the safety of honey from Africa and some baseline information for future studies on other components of the hive matrix in relation to honey bee colony losses.

\section{Methods}

\section{Chemicals and reagents}

All pesticide standards were of high purity $(>94 \%)$ and were obtained from Sigma-Aldrich (Chemie $\mathrm{GmbH}$, Germany) and Dr Ehrenstorfer (Augsburg, Germany) and were stored according to manufacturer's recommendations until use. Pesticide stock solutions were prepared in acetonitrile at $1 \mu \mathrm{g} / \mathrm{mL}$ and stored in amber screw-capped glass vials at $-20{ }^{\circ} \mathrm{C}$.

\section{LC-MS/MS instrumentation}

An Agilent 1290 ultra high performance liquid chromatography (UHPLC) series coupled to a 6490 model triple quadrupole mass spectrometer (Agilent technologies) with an ifunnel JetStream electrospray source operating in the positive ionization mode was applied using dynamic multi-reaction monitoring (DMRM) software features. The electrospray ionization settings were gas temperature, $120{ }^{\circ} \mathrm{C}$; gas flow, $15 \mathrm{~L} / \mathrm{min}$; nebuliser gas, $30 \mathrm{psi}$; sheath gas temperature, $375^{\circ} \mathrm{C}$; sheath gas flow, $12 \mathrm{~L} / \mathrm{min}$; capillary voltage, $3500 \mathrm{~V}$; nozzle voltage, $300 \mathrm{~V}$. The ifunnel parameters were high pressure RF $150 \mathrm{~V}$ and low pressure RF $60 \mathrm{~V}$. Nitrogen was used both as a nebuliser and as the collision gas. Mass Hunter Data Acquisition; Qualitative and Quantitative analysis 
software (Agilent Technologies, Palo Alto, CA, v.B.06 and v.B.07) were used for method development, data acquisition and data processing for all the analyses.

The chromatographic separation was performed on a Rapid Resolution reverse phase column-C18 $1.8 \mu \mathrm{m}$, $2.1 \times 150 \mathrm{~mm}$ column (Agilent Technologies). The mobile phases comprised of $100 \%$ water in $5 \mathrm{mM}$ ammonium formate containing $0.1 \%$ formic acid for solvent $\mathrm{A}$ and acetonitrile in $5 \mathrm{mM}$ ammonium formate containing $0.1 \%$ formic acid for solvent B. A gradient elution at a flow rate of $0.4 \mathrm{~mL} / \mathrm{min}$ was used.

\section{Optimization of LC-MS/MS parameters}

Pesticide standard solutions, individually or as mixes, were used for method development and instrument parameters optimization. To ensure that the maximum sensitivity for identification and quantification of the targeted pesticides is obtained, careful optimization of all MS parameters was performed by infusing the standard solutions directly into the MS followed by infusion through the column to establish their respective retention times (RT). The parameters optimised included collision energy (CE), gas temperature; gas flow, sheath gas temperature and flow, high and low pressure radio-frequency. Table 1 demonstrates the parameters developed and optimised for the 96 pesticide residues targeted in this study.

\section{Data analysis}

Targeted analytes were identified by monitoring two transition ions where possible, for each analyte as recommended by SANCO guidelines for LC-MS/MS analysis (SANCO/12571/2013). The most dominant transition ion was used for quantification whereas the second most intense ion as a qualifier for confirmation purposes. Calibration standard solutions were prepared at seven calibration levels covering a concentration range of 0.1 to 100 parts per billion (ppb), including the zero point. The resulting calibration curve was used to determine the instrument's limit of reporting (LOR) and limits of detection (LOD). These were set as calibration standard concentrations producing signal to noise ratio of 3 and 10 respectively. The LOR was set as the minimum concentration that could be quantified with acceptable accuracy and precision. The LC-MS/MS system's linearity was evaluated by assessing the signal responses of the calibration standards.

\section{Sample preparation}

Prior analysis of a honey sample, obtained from the local organic farmer from Kenya, was performed to ensure that it did not contain any of the studied compounds. This sample was selected as a blank during method development for spiking, preparing matrix matched calibration curves and recovery purposes. Samples were prepared following the QuEChERS method (Anastassiades et al. 2003) with some modifications. Briefly, $5 \mathrm{~g}$ of this sample was weighed into a $50 \mathrm{ml}$ falcon tube and $10 \mathrm{ml}$ of water were added and the mixture homogenized. Acetonitrile $(10 \mathrm{ml})$ plus a mixture of salts $(4 \mathrm{~g}$ magnesium sulphate, $1 \mathrm{~g}$ sodium chloride, $1 \mathrm{~g}$ of trisodium citrate dehydrate and $0.5 \mathrm{~g}$ of disodium hydrogen citrate sesquihydrate) were added and the samples were vortexed for $1 \mathrm{~min}$ and centrifuged at $4200 \mathrm{rpm}$ for $5 \mathrm{~min}$. Aliquots of the supernatant were transferred to separate eppendorf tubes and subjected to either no clean-up or to various QuEChERS clean-up methods. A portion of $1 \mathrm{~mL}$ of the final solution was then transferred to an auto-sampler vial for LC-MS/MS analysis.

\section{Extraction efficiency}

A series of spiked samples were used to assess extraction efficiency of the method. These samples were prepared as follows: blank honey samples fortified at 10 times LOQ (10 ng/g) were dissolved in appropriate amounts of water and homogenized. Extractions of the spiked residues were performed following QuEChERS methods. Honey samples were spiked with a mixture of pesticide residues possessing different physic-chemical properties. After extraction, aliquots of the extract were subjected to three QuEChERS clean-up methods (PSA plus GCB or PSA plus C18 or PSA alone). Figure 1 represents a schematic diagram illustrating the workflow that was employed during method development. Extraction efficiencies of these clean-up methods were compared to extraction efficiencies of no clean-up methods to evaluate which of those methods will be best suited for our analysis. Instead, these samples were subjected to high centrifugation $\left(12,000 \mathrm{rpm}\right.$ held at $4{ }^{\circ} \mathrm{C}$ ) for $10 \mathrm{~min}$ and filtered through $0.22 \mu \mathrm{m}$ PTFE filters on a Samplicity system (Merck Millipore, Germany). Each test was replicated three times.

\section{Matrix effects}

The effect of matrix co-extractives was performed by assessing ion suppression or enhancement effects of signals from chromatograms of matrix matched standard solutions compared to spiked extracts at the same concentration levels as per DG SANCO guidelines for LC-MS/MS analysis (SANCO/12571/2013). These were prepared using the extract of blank matrix (honey) covering a target analyte concentration range of 0.1 to $100 \mathrm{ng} / \mathrm{g}$. Detection and quantification limits of the method were determined as described previously.

\section{Validation of the analytical procedure}

Analytes to be validated were spiked into the blank honey sample at LOR $(1 \mathrm{ng} / \mathrm{g})$ and at the lowest MRL level $(0.01 \mathrm{mg} / \mathrm{kg}$ or $10 \mathrm{ng} / \mathrm{g})$. Analysis was performed as 
Table 1 Instrumental parameters of the MS/MS detector and retention times (RT) of the 96 pesticides standard mixture used for method development

\begin{tabular}{|c|c|c|c|c|c|c|}
\hline Compound name & RT (min) & Parent ion $(m / z)$ & ${ }^{\mathrm{a}}$ Trans1 & CE1(V) & ${ }^{\mathrm{a}}$ Trans2 & CE2(V) \\
\hline Omethoate & 2.72 & 214 & 125 & 20 & 109 & 25 \\
\hline Acetamiprid & 2.84 & 223 & 126 & 20 & 90 & 35 \\
\hline Acephate & 2.84 & 184 & 143 & 5 & 125 & 15 \\
\hline Propamocarb & 3.19 & 189 & 144 & 5 & 102 & 15 \\
\hline Oxamyl & 3.58 & 237 & 90 & 0 & 72 & 15 \\
\hline Methomyl & 3.84 & 163 & 106 & 5 & 88 & 0 \\
\hline Thiamethoxam & 3.95 & 292 & 211 & 5 & 181 & 20 \\
\hline Monocrotophos & 3.95 & 224 & 193 & 0 & 127 & 10 \\
\hline Aldicarb & 3.98 & 208 & 116 & 0 & 89 & 10 \\
\hline Imidacloprid & 4.42 & 256 & 209 & 10 & 175 & 15 \\
\hline Thiabendazol & 4.45 & 202 & 175 & 25 & 131 & 35 \\
\hline Cymiazole & 4.70 & 219 & 171 & 25 & 144 & 35 \\
\hline Dimethoate & 4.82 & 230 & 199 & 0 & 125 & 20 \\
\hline Thiacloprid & 5.13 & 253 & 126 & 20 & 90 & 40 \\
\hline Propagite & 5.25 & 368 & 231 & 5 & 175 & 10 \\
\hline Aldicarb fragment & 5.43 & 116 & 89 & 4 & 70 & 4 \\
\hline Pirimicarb & 5.90 & 239 & 182 & 10 & 72 & 20 \\
\hline Dichlorvos & 6.13 & 221 & 109 & 12 & 79 & 24 \\
\hline Carbofuran & 6.36 & 222 & 165 & 5 & 123 & 20 \\
\hline Nicosulfuron & 6.40 & 411 & 213 & 12 & 182 & 16 \\
\hline Metsulfuron-methyl & 6.51 & 382 & 199 & 20 & 167 & 15 \\
\hline Metribuzin & 6.54 & 215 & 187 & 15 & 84 & 20 \\
\hline Malathion & 6.64 & 331 & 126 & 5 & 99 & 10 \\
\hline Carbaryl & 6.93 & 202 & 145 & 0 & 127 & 25 \\
\hline Fosthiazole & 7.16 & 284 & 228 & 5 & 104 & 20 \\
\hline Thiodicarb & 7.16 & 355 & 108 & 10 & 88 & 10 \\
\hline Amidosulfuron & 7.22 & 370 & 261 & 10 & 218 & 20 \\
\hline DEET & 7.75 & 192 & 119 & 16 & 91 & 32 \\
\hline Molinate & 7.75 & 188 & 126 & 25 & 98 & 12 \\
\hline Tribenuron-methyl & 7.87 & 396 & 155 & 5 & & \\
\hline Metalaxyl & 7.89 & 280 & 220 & 10 & 160 & 20 \\
\hline Flutriafol & 8.01 & 302 & 70 & 15 & 123 & 30 \\
\hline Diuron & 8.02 & 233 & 72 & 20 & 72 & 20 \\
\hline Isoxafluote & 8.08 & 360 & 251 & 20 & 220 & 35 \\
\hline Methidathion & 8.46 & 303 & 145 & 0 & 85 & 15 \\
\hline Flazasulfuron & 8.73 & 408 & 182 & 15 & & \\
\hline Fenobucarb & 8.79 & 208 & 152 & 5 & 95 & 10 \\
\hline Azoxystrobin & 9.01 & 404 & 372 & 10 & 344 & 25 \\
\hline Linuron & 9.19 & 249 & 182 & 10 & 160 & 15 \\
\hline Fludioxonil & 9.30 & 247 & 169 & 32 & 126 & 32 \\
\hline Promecarb & 9.64 & 208 & 151 & 0 & & \\
\hline Bosclid & 9.67 & 343 & 271 & 28 & 307 & 12 \\
\hline Triadimefon & 10.01 & 294 & 197 & 10 & 69 & 20 \\
\hline
\end{tabular}


Table 1 Instrumental parameters of the MS/MS detector and retention times (RT) of the 96 pesticides standard mixture used for method development (Continued)

\begin{tabular}{|c|c|c|c|c|c|c|}
\hline Bromuconazole & 10.02 & 378 & 159 & 35 & 70 & 20 \\
\hline Bifenazate & 10.09 & 301 & 170 & 15 & & \\
\hline Cyproconazole & 10.16 & 292 & 70 & 15 & 125 & 35 \\
\hline Fluquinconazole & 10.27 & 376 & 349 & 16 & 307 & 24 \\
\hline Iprovalicarb & 10.27 & 321 & 203 & 0 & 119 & 20 \\
\hline Triadimenol & 10.36 & 296 & 70 & 5 & 99 & 10 \\
\hline Flufenacet & 10.38 & 364 & 194 & 5 & 152 & 15 \\
\hline Bupirimate & 10.42 & 317 & 166 & 20 & 108 & 25 \\
\hline Tetraconazole & 10.45 & 372 & 159 & 30 & 70 & 20 \\
\hline Ethoprophos & 10.48 & 243 & 131 & 15 & 97 & 30 \\
\hline Epoxyconazol & 10.65 & 330 & 121 & 20 & 101 & 45 \\
\hline Cyazofamid & 10.68 & 325 & 261 & 5 & 108 & 10 \\
\hline Cyprodinil & 10.81 & 226 & 93 & 40 & 77 & 45 \\
\hline Fenbuconazole & 10.85 & 337 & 125 & 35 & 70 & 15 \\
\hline Metolachlor & 10.94 & 284 & 252 & 10 & 176 & 20 \\
\hline Fenamiphos & 10.95 & 304 & 217 & 20 & 202 & 35 \\
\hline Flusilazole & 10.97 & 316 & 247 & 15 & 165 & 25 \\
\hline Picoxystrobin & 11.05 & 368 & 205 & 0 & 145 & 20 \\
\hline Tebufenozid & 11.10 & 353 & 297 & 0 & 133 & 15 \\
\hline Diflubenzuron & 11.17 & 311 & 158 & 10 & 141 & 35 \\
\hline Rotenone & 11.24 & 395 & 213 & 20 & 192 & 20 \\
\hline Fipronil & 11.25 & 435 & 330 & 12 & 250 & 28 \\
\hline Kresoxim-methyl & 11.53 & 314 & 267 & 0 & 222 & 10 \\
\hline Tebuconazole & 11.53 & 308 & 125 & 40 & 70 & 20 \\
\hline Procymidon & 11.64 & 284 & 67 & 12 & 256 & 28 \\
\hline Benalaxyl & 11.71 & 326 & 294 & 5 & 148 & 15 \\
\hline Diazinon & 11.71 & 305 & 169 & 20 & 153 & 20 \\
\hline Coumaphos & 11.76 & 363 & 307 & 16 & 227 & 28 \\
\hline Prochloraz & 11.76 & 376 & 308 & 5 & 266 & 10 \\
\hline Chlorfenvinphos & 11.77 & 359 & 170 & 40 & 155 & 8 \\
\hline Hexaconazole & 11.93 & 314 & 159 & 30 & 70 & 15 \\
\hline Pyraclostrobin & 12.04 & 388 & 194 & 5 & 163 & 20 \\
\hline Clofentezin & 12.06 & 303 & 138 & 10 & 102 & 40 \\
\hline Pirimiphos-methyl & 12.21 & 306 & 164 & 20 & 108 & 30 \\
\hline Spinosyn A & 12.23 & 732 & 142 & 30 & 98 & 45 \\
\hline Metconazole & 12.30 & 320 & 125 & 40 & & \\
\hline Bitertenol & 12.38 & 338 & 269 & 0 & 70 & 0 \\
\hline Chlorpyrifos-methyl & 12.41 & 322 & 290 & 10 & 125 & 25 \\
\hline Trifloxystrobin & 12.78 & 409 & 186 & 10 & 145 & 45 \\
\hline Spinosyn D & 12.88 & 747 & 142 & 35 & 98 & 55 \\
\hline Ipconazole & 12.97 & 334 & 125 & 45 & 70 & 25 \\
\hline Indoxacarb & 12.99 & 528 & 203 & 45 & 150 & 20 \\
\hline Novaluron & 13.32 & 493 & 158 & 20 & 141 & 45 \\
\hline Buprofezin & 13.45 & 306 & 201 & 5 & 116 & 10 \\
\hline
\end{tabular}


Table 1 Instrumental parameters of the MS/MS detector and retention times (RT) of the 96 pesticides standard mixture used for method development (Continued)

\begin{tabular}{|c|c|c|c|c|c|c|}
\hline Profenofos & 13.48 & 375 & 347 & 5 & 305 & 15 \\
\hline Ethion & 13.93 & 385 & 199 & 4 & 143 & 20 \\
\hline Temephos & 14.02 & 467 & 419 & 20 & 125 & 44 \\
\hline Chlorpyrifos & 14.08 & 350 & 200 & 15 & 198 & 15 \\
\hline Pyriproxyfen & 14.17 & 322 & 185 & 20 & 96 & 10 \\
\hline Lufenuron & 14.19 & 511 & 158 & 20 & 141 & 45 \\
\hline Hexythiazox & 14.46 & 353 & 228 & 10 & 168 & 25 \\
\hline Fenazaquin & 15.35 & 307 & 161 & 10 & 57 & 25 \\
\hline Pyridaben & 15.44 & 365 & 309 & 10 & 147 & 25 \\
\hline Bifenthrin & 16.47 & 440 & 181 & 5 & 166 & 20 \\
\hline Etofenprox & 16.57 & 394 & 177 & 10 & 107 & 45 \\
\hline
\end{tabular}

${ }^{\mathrm{a}}$ Transition ions used to quantify and qualify the targeted analytes

described previously. The recoveries and precision of the extraction method were determined as the average of five replicates. The method linearity was evaluated by assessing the signal responses of the targeted analytes from matrix-matched calibration solutions prepared by spiking blank extracts at seven concentration levels,

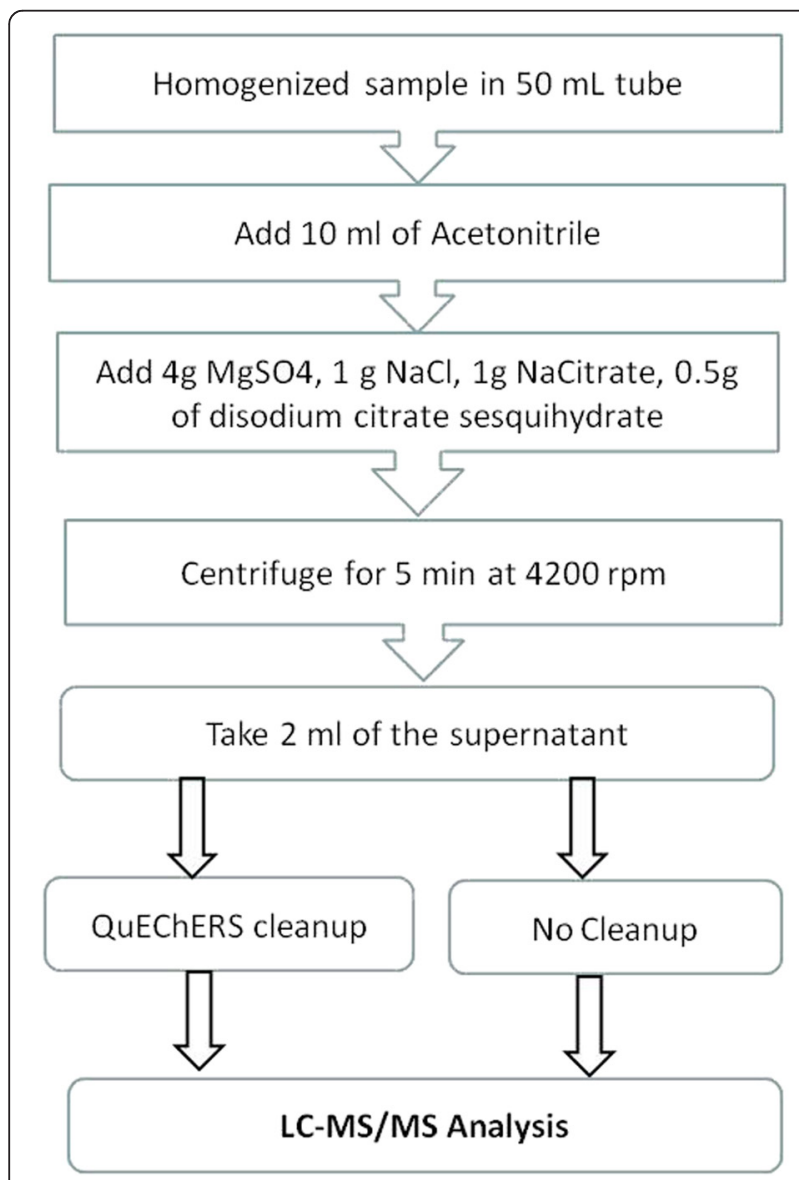

Fig. 1 Schematic diagram representing sample preparation workflow from 0.1 to $100 \mathrm{ng} / \mathrm{g}$, including the zero point or the blank. The method precision was expressed as percent relative standard deviation (\%RSD) of the intra-day and inter-day analyses $(n=5)$. Blank matrices along with reagent blank were run during validation to ensure minimal risk of interferences, guarantee specificity of the method and to check for potential solvent contamination.

\section{Application to real samples}

The developed method was applied to conduct a preliminary study on chemical contaminants present in commercial honey in Africa. Ethiopia and Kenya were selected for this study as they are among the major producers of honey in Africa. From each country, 14 commercial honey samples were collected from local markets/farmers. These samples consisted of five honey samples from stingless (Apis meliponina) and nine honey bee (Apis mellifera) samples from various regions in each country. A total of 28 samples were analyzed at the African Reference Laboratory for Bee Health, International Centre of Insect Physiology and Ecology (icipe), Duduville Campus, Nairobi, Kenya at two different seasons (November 2014 and July 2015). All samples were stored in their original packaging under the recommended conditions prior to use and were prepared as previously described. The same calibration curve described above was run at the end of the sample series to check the stability of the detector after data acquisition of the unknown samples.

\section{Statistical analysis}

Data were analyzed using $\mathrm{R}$ version 3.1.1 ( $\mathrm{R}$ Core Team 2014). For each pesticide or compound, the four cleanup methods were compared using one-way Analysis of Variance (ANOVA) and the means separated using the Student-Newman-Kuels (SNK) test. All tests were performed at $5 \%$ significance level. Means with the same letter across are not significantly different. 


\section{Results and discussion LC-MS/MS analysis}

In this study, the methods investigated were selected based on the known matrix interferences expected from honey. Since sugars constitute the greatest proportion of honey $(>75 \%)$, three of the four methods investigated included PSA, as it removes sugars, along other interferences. Samples were spiked with a mixture of 96 pesticide standards at the default MRL value $(0.01 \mathrm{mg} / \mathrm{kg})$ since it provided great recoveries with the best reproducibility across multiple analytes during method development. Figure 2 shows representative chromatograms of honey extract processed using the four clean-up methods. Although the chromatographic profiles appeared similar for the four clean-up methods, the lowest recoveries were obtained from pesticides subjected to PSA combined with GCB clean-up with recoveries ranging from 5 to $117 \%$ (Table 2). The use of GCB was important in removing pigment in honey; however, it also resulted in significant analyte losses during sample clean-up which could potentially lead to false negative results. Out of the 96 pesticides evaluated, 51 pesticides had the lowest recoveries from this method compared to the other methods (Table 2). Additionally, more than $45 \%$ of the pesticides subjected to this method did not meet the minimum recommended criteria (>70 \%) as indicated in the Guidance document on analytical quality control and validation procedures for pesticide residues analysis in food and feed (SANCO/ 12571/2013). On the other hand, for most pesticides, the best recoveries were obtained when PSA was used as a clean-up method. When compared to PSA plus C18 clean-up method, there were significant $(P<0.05)$ differences in more than $10 \%$ of the pesticides evaluated.
Results from this study also indicate that out of the 96 pesticides studied, only three pesticides, nicosulfuron (43\%), procymidon (58 \%) and propamocarb (58\%), had recoveries that were below the acceptable limit when PSA was used alone. There was no significant $(P<0.05)$ difference in recoveries for procymidon cleaned using $\mathrm{C} 18$ plus PSA (78 \%) and PSA alone (58 \%). Therefore, to improve recoveries for nicosulfuron and propamocarb, other alternatives must be considered. For instance, for nicosulfuron, based on the data provided in Table 2, the clean-up step can be omitted to yield $100 \%$ recovery. This suggests that in the absence of clean-up resources, satisfactory information on levels of residue contamination in honey can still be achieved with minimal sample manipulations as found in other studies (Kujawski et al. 2014). Although omitting the clean-up step offers time savings in sample processing and is more economical, further precaution must be taken to avoid any potential clogging of the LC-MS system or eventual contamination of the MS ionization source. Based on the findings highlighted in Table 2, the use of PSA was selected as the best method for our analysis but was complemented with the no clean-up method to maximize on recoveries of all targeted pesticides.

Analytes eluted in $17 \mathrm{~min}$ followed by a short highorganic rinse to maintain the column and also in avoiding matrix carryover into the next sample. Elution of the remaining matrix material during subsequent analysis can cause unexpected matrix effects resulting in significant ionization inefficiencies. Matrix effects may either result to signal enhancement leading to recoveries $>100 \%$ or signal suppression resulting in poor recoveries. Aside from polar pesticides, other pesticides were well distributed across the elution window facilitating proper scan rate for scheduled

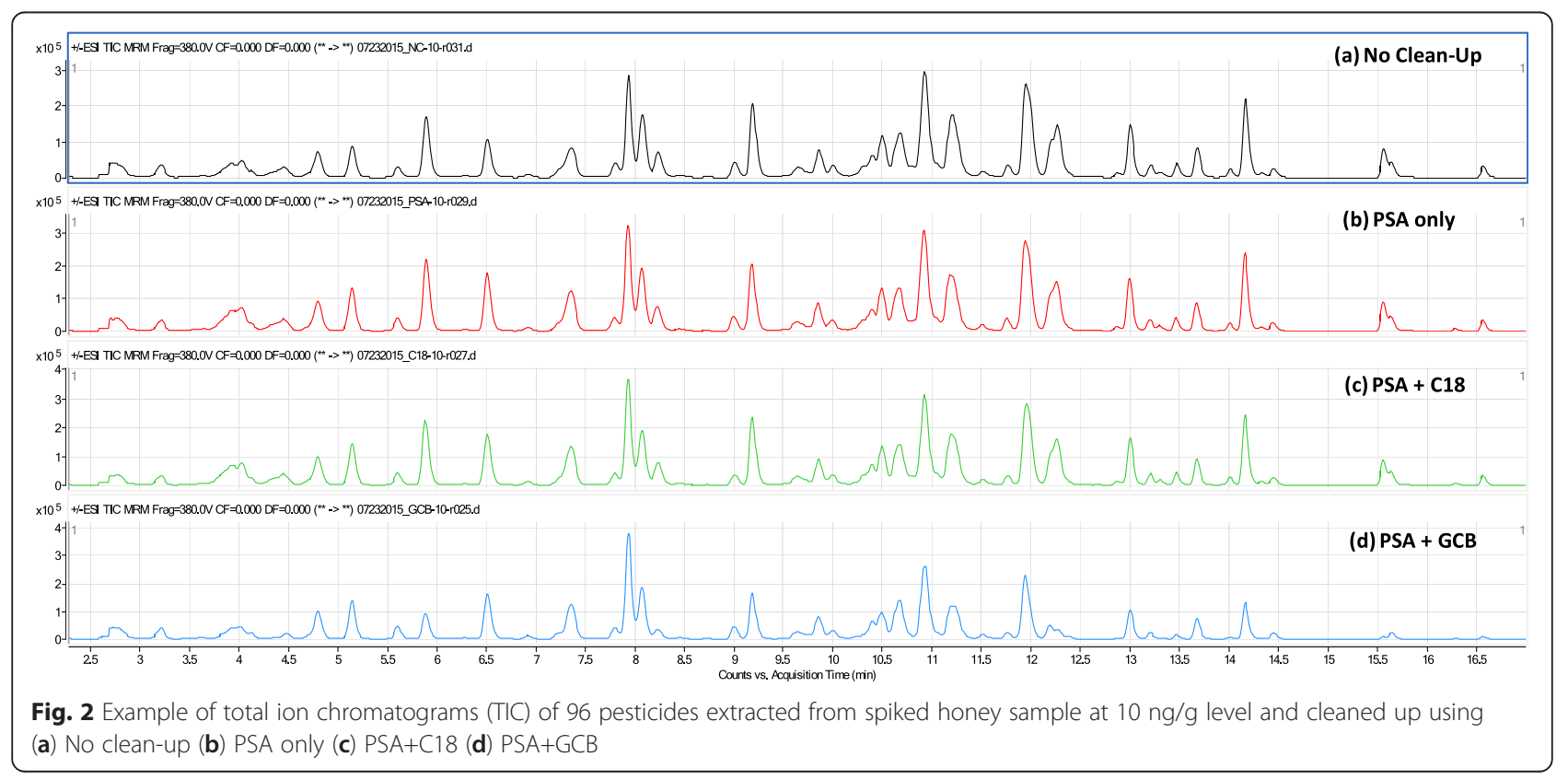


Table 2 Percentage recoveries ( \pm SD) of 96 pesticides subjected to either QuEChERS clean-up methods or no clean-up

\begin{tabular}{|c|c|c|c|c|}
\hline \multirow[b]{2}{*}{ Compound name } & \multicolumn{4}{|c|}{$\%$ recovery at $10 \mathrm{LOR}(10 \mathrm{ng} / \mathrm{g}) \pm \mathrm{SD}$} \\
\hline & GCB+PSA & C18+PSA & PSA & No clean-up \\
\hline Acephate & $72.8 \pm 0.8^{(b)}$ & $85.1 \pm 0.6^{(a)}$ & $76.1 \pm 0.8^{(a b)}$ & $52.5 \pm 0.7^{(\mathrm{c})}$ \\
\hline Acetamiprid & $98.1 \pm 0.6^{(a)}$ & $99.8 \pm 0.03^{(a)}$ & $99.6 \pm 0.3^{(\mathrm{a})}$ & $74.8 \pm 0.0^{(b)}$ \\
\hline Aldicarb fragment & $104.5 \pm 0.4^{(\mathrm{a})}$ & $100.5 \pm 0.3^{(b)}$ & $97.9 \pm 0.2^{(b)}$ & $70.6 \pm 0.1^{(\mathrm{c})}$ \\
\hline Amidosulfuron & $87.0 \pm 0.8^{(b)}$ & $74.3 \pm 0.4^{(\mathrm{c})}$ & $89.5 \pm 0.1^{(b)}$ & $94.3 \pm 0.2^{(a)}$ \\
\hline Azoxystrobin & $77.0 \pm 0.7^{(b)}$ & $108.8 \pm 0.8^{(\mathrm{a})}$ & $106.5 \pm 0.8^{(\mathrm{a})}$ & $101.8 \pm 0.5^{(a)}$ \\
\hline Benalaxyl & $88.9 \pm 0.6^{(a)}$ & $97.3 \pm 1.0^{(\mathrm{a})}$ & $97.5 \pm 0.4^{(\mathrm{a})}$ & $97.7 \pm 0.6^{(a)}$ \\
\hline Bifenazate & $23.7 \pm 0.8^{(b)}$ & $117.5 \pm 0.3^{(a)}$ & $111.6 \pm 1.2^{(\mathrm{a})}$ & $121.6 \pm 0.4^{(a)}$ \\
\hline Bifenthrin & $45.7 \pm 1.1^{(\mathrm{b})}$ & $92.5 \pm 0.9^{(a)}$ & $79.8 \pm 0.8^{(\mathrm{a})}$ & $90.2 \pm 0.02^{(a)}$ \\
\hline Bitertanol & $88.9 \pm 0.6^{(\mathrm{b})}$ & $105.6 \pm 0.4^{(\mathrm{a})}$ & $99.4 \pm 0.01^{(\mathrm{a})}$ & $100.6 \pm 0.7^{(a, b)}$ \\
\hline Bosclid (Nicobifen) & $39.6 \pm 1.0^{(b)}$ & $113.1 \pm 1.3^{(\mathrm{a})}$ & $106.3 \pm 0.4^{(\mathrm{a})}$ & $115.3 \pm 0.7^{(\mathrm{a})}$ \\
\hline Bromuconazole & $85.2 \pm 1.6^{(\mathrm{b})}$ & $96.9 \pm 0.1^{(a b)}$ & $103.0 \pm 0.4^{(\mathrm{a})}$ & $92.3 \pm 0.4^{(\mathrm{ab})}$ \\
\hline Bupirimate & $61.3 \pm 0.6^{(\mathrm{b})}$ & $104.6 \pm 0.1^{(a)}$ & $102.5 \pm 0.6^{(\mathrm{a})}$ & $110.7 \pm 1.4^{(\mathrm{a})}$ \\
\hline Buprofezin & $84.6 \pm 0.5^{(c)}$ & $104.0 \pm 0.4^{(\mathrm{ab})}$ & $106.9 \pm 0.4^{(\mathrm{a})}$ & $102.9 \pm 0.8^{(b)}$ \\
\hline Carbaryl & $98.2 \pm 1.2^{(a)}$ & $110.9 \pm 0.9^{(a)}$ & $102.5 \pm 0.2^{(\mathrm{a})}$ & $72.6 \pm 0.1^{(\mathrm{b})}$ \\
\hline Carbofuran & $108.3 \pm 0.8^{(b)}$ & $120.4 \pm 0.9^{(a)}$ & $119.9 \pm 0.1^{(\mathrm{ab})}$ & $64.1 \pm 0.6^{(c)}$ \\
\hline Chlorfenvinphos & $78.1 \pm 0.7^{(\mathrm{c})}$ & $93.6 \pm 0.1^{(b)}$ & $103.6 \pm 0.2^{(a)}$ & $94.9 \pm 0.7^{(b)}$ \\
\hline Chlorpyrifos & $21.4 \pm 0.6^{(\mathrm{b})}$ & $93.4 \pm 0.6^{(a)}$ & $94.2 \pm 0.3^{(a)}$ & $87.6 \pm 1.0^{(a)}$ \\
\hline Chlorpyrifos-methyl & $26.4 \pm 0.8^{(c)}$ & $105.3 \pm 0.5^{(\mathrm{a})}$ & $99.5 \pm 0.1^{(\mathrm{a})}$ & $95.5 \pm 0.4^{(b)}$ \\
\hline Clofentezin & $6.2 \pm 0.7^{(b)}$ & $97.3 \pm 0.3^{(a)}$ & $98.5 \pm 0.3^{(a)}$ & $91.1 \pm 0.8^{(a)}$ \\
\hline Coumaphos & $5.4 \pm 0.4^{(b)}$ & $102.6 \pm 1.3^{(\mathrm{a})}$ & $105.2 \pm 0.5^{(\mathrm{a})}$ & $109.2 \pm 0.5^{(a)}$ \\
\hline Cyazofamid & $79.1 \pm 0.2^{(c)}$ & $102.2 \pm 0.1^{(a)}$ & $100.6 \pm 0.2^{(a)}$ & $92.3 \pm 0.2^{(b)}$ \\
\hline Cymiazol & $56.0 \pm 0.9^{(c)}$ & $92.2 \pm 0.4^{(a)}$ & $89.5 \pm 0.1^{(\mathrm{a})}$ & $75.2 \pm 0.5^{(\mathrm{a})}$ \\
\hline Cyproconazole & $100.3 \pm 0.2^{(b)}$ & $87.8 \pm 1.0^{(b)}$ & $106.8 \pm 0.2^{(a)}$ & $92.3 \pm 0.5^{(\mathrm{b})}$ \\
\hline Cyprodinil & $10.5 \pm 0.6^{(\mathrm{c})}$ & $90.8 \pm 0.2^{(b)}$ & $104.5 \pm 0.4^{(\mathrm{a})}$ & $105.0 \pm 1.0^{(\mathrm{ab})}$ \\
\hline DEET & $109.1 \pm 0.4^{(\mathrm{a})}$ & $100.4 \pm 1.2^{(\mathrm{a})}$ & $96.0 \pm 0.0^{(\mathrm{a})}$ & $83.5 \pm 0.5^{(\mathrm{b})}$ \\
\hline Diazinon & $81.4 \pm 0.01^{(b)}$ & $98.7 \pm 0.3^{(a)}$ & $99.0 \pm 0.3^{(a)}$ & $99.4 \pm 1.1^{(\mathrm{a})}$ \\
\hline Dichlorvos & $107.0 \pm 0.8^{(a)}$ & $97.3 \pm 0.6^{(b)}$ & $99.4 \pm 0.3^{(\mathrm{b})}$ & $85.7 \pm 0.2^{(c)}$ \\
\hline Diflubenzuron & $18.9 \pm 4.8^{(\mathrm{b})}$ & $101.9 \pm 0.3^{(\mathrm{a})}$ & $106.3 \pm 1^{(\mathrm{a})}$ & $104.8 \pm 0.6^{(a)}$ \\
\hline Dimethoate & $99.2 \pm 1.0^{(\mathrm{a})}$ & $101.7 \pm 0.2^{(\mathrm{a})}$ & $94.3 \pm 0.3^{(a)}$ & $62.8 \pm 0.1^{(b)}$ \\
\hline Diuron & $33.0 \pm 0.8^{(c)}$ & $100.7 \pm 0.6^{(a)}$ & $108.1 \pm 0.2^{(\mathrm{a})}$ & $92.1 \pm 0.4^{(b)}$ \\
\hline Epoxyconazol & $38.8 \pm 2.6^{(b)}$ & $91.2 \pm 0.2^{(a)}$ & $96.4 \pm 1.1^{(\mathrm{a})}$ & $89.9 \pm 0.6^{(a)}$ \\
\hline Ethion & $78.7 \pm 0.2^{(b)}$ & $98.3 \pm 0.1^{(\mathrm{a})}$ & $103.0 \pm 0.5^{(\mathrm{a})}$ & $95.1 \pm 0.1^{(a)}$ \\
\hline Ethoprophos & $87.7 \pm 0.9^{(a)}$ & $94.1 \pm 0.4^{(\mathrm{a})}$ & $98.3 \pm 1.0^{(\mathrm{a})}$ & $90.8 \pm 0.7^{(a)}$ \\
\hline Etofenprox & $24.2 \pm 0.5^{(\mathrm{b})}$ & $98.5 \pm 0.4^{(a)}$ & $99.5 \pm 0.1^{(\mathrm{a})}$ & $92.2 \pm 0.0^{(a)}$ \\
\hline Fenamiphos & $56.4 \pm 1.0^{(\mathrm{b})}$ & $107.8 \pm 0.2^{(\mathrm{a})}$ & $111.6 \pm 0.3^{(\mathrm{a})}$ & $107.8 \pm 0.5^{(\mathrm{a})}$ \\
\hline Fenazaquin & $9.9 \pm 1.7^{(d)}$ & $93.5 \pm 0.5^{(\mathrm{b})}$ & $98.4 \pm 0.1^{(a)}$ & $89.4 \pm 0.1^{(\mathrm{c})}$ \\
\hline Fenbuconazole & $40.5 \pm 1.2^{(b)}$ & $107.8 \pm 0.3^{(\mathrm{a})}$ & $109.1 \pm 0.9^{(\mathrm{a})}$ & $107.0 \pm 0.3^{(\mathrm{a})}$ \\
\hline Fenobucarb & $94.7 \pm 2.0^{(\mathrm{b})}$ & $80.6 \pm 0.4^{(c)}$ & $101.6 \pm 0.1^{(\mathrm{a})}$ & $90.4 \pm 0.2^{(b)}$ \\
\hline Fipronil & $107.9 \pm 1.0^{(\mathrm{a})}$ & $111.4 \pm 0.1^{(\mathrm{a})}$ & $108.3 \pm 0.3^{(\mathrm{a})}$ & $111.1 \pm 0.4^{(\mathrm{a})}$ \\
\hline Flazasulfuron & $81.1 \pm 1.5^{(\mathrm{b})}$ & $46.7 \pm 0.5^{(\mathrm{d})}$ & $70.3 \pm 0.3^{(c)}$ & $96.7 \pm 0.4^{(a)}$ \\
\hline Fludioxonil & $34.1 \pm 2.9^{(\mathrm{b}}$ & $105.9 \pm 0.5^{(\mathrm{a})}$ & $104.7 \pm 0.1^{(a)}$ & $110.8 \pm 0.5^{(\mathrm{a})}$ \\
\hline Flufenacet & $102.8 \pm 1.9^{(a)}$ & $117.5 \pm 0.9^{(\mathrm{a})}$ & $103.8 \pm 0.7^{(\mathrm{a})}$ & $100.9 \pm 0.5^{(a)}$ \\
\hline Fluquinconazole & $43.6 \pm 2.0^{(\mathrm{b})}$ & $92.7 \pm 1.1^{(\mathrm{a})}$ & $99.3 \pm 0.9^{(a)}$ & $90.5 \pm 0.9^{(a)}$ \\
\hline
\end{tabular}


Table 2 Percentage recoveries $( \pm \mathrm{SD}$ ) of 96 pesticides subjected to either QuEChERS clean-up methods or no clean-up (Continued)

\begin{tabular}{|c|c|c|c|c|}
\hline Flusilazole & $97.8 \pm 0.8^{(\mathrm{b})}$ & $117.4 \pm 0.7^{(a)}$ & $108.3 \pm 0.8^{(\mathrm{ab})}$ & $98.6 \pm 0.5^{(\mathrm{ab})}$ \\
\hline Flutriafol & $94.3 \pm 0.2^{(\mathrm{b})}$ & $97.9 \pm 0.4^{(\mathrm{ab})}$ & $101.3 \pm 0.1^{(a)}$ & $96.8 \pm 0.5^{(\mathrm{ab})}$ \\
\hline Fosthiazate & $101.8 \pm 0.2^{(a)}$ & $107.1 \pm 0.9^{(a)}$ & $103.6 \pm 0.1^{(\mathrm{a})}$ & $70.1 \pm 0.1^{(b)}$ \\
\hline Hexaconazole & $90.0 \pm 1.0^{(\mathrm{b})}$ & $99.3 \pm 1.1^{(\mathrm{b})}$ & $110.5 \pm 0.5^{(\mathrm{a})}$ & $97.9 \pm 0.1^{(b)}$ \\
\hline Hexythiazox & $77.2 \pm 0.6^{(c)}$ & $99.8 \pm 0.1^{(a)}$ & $99.6 \pm 0.2^{(\mathrm{a})}$ & $94.1 \pm 0.4^{(b)}$ \\
\hline Imidacloprid & $80.3 \pm 0.2^{(a)}$ & $88.3 \pm 0.2^{(\mathrm{a})}$ & $87.6 \pm 0.3^{(a)}$ & $66.4 \pm 0.3^{(b)}$ \\
\hline Indoxacarb & $56.4 \pm 1.6^{(b)}$ & $103.0 \pm 0.5^{(a)}$ & $102.1 \pm 0.1^{(a)}$ & $96.1 \pm 0.3^{(a)}$ \\
\hline Ipconazole & $57.7 \pm 0.9^{(\mathrm{b})}$ & $103.7 \pm 0.2^{(a)}$ & $102.7 \pm 0.1^{(\mathrm{a})}$ & $98.9 \pm 0.5^{(a)}$ \\
\hline Iprovalicarb & $58.6 \pm 6.5^{(a)}$ & $95.6 \pm 0.1^{(\mathrm{a})}$ & $99.1 \pm 1.3^{(\mathrm{a})}$ & $74.5 \pm 1.2^{(a)}$ \\
\hline Isoxaflutole & $99.0 \pm 0.3^{(a)}$ & $98.9 \pm 0.5^{(\mathrm{a})}$ & $105.6 \pm 0.4^{(a)}$ & $120.8 \pm 2.0^{(a)}$ \\
\hline Kresoxim-methyl & $74.7 \pm 0.2^{(a)}$ & $96.0 \pm 1.1^{(\mathrm{a})}$ & $93.1 \pm 0.3^{(\mathrm{a})}$ & $92.3 \pm 0.8^{(a)}$ \\
\hline Linuron & $39.7 \pm 3.4^{(\mathrm{b})}$ & $103.3 \pm 0.03^{(a)}$ & $107.9 \pm 0.5^{(\mathrm{a})}$ & $97.5 \pm 0.1^{(\mathrm{a})}$ \\
\hline Lufenuron & $5.9 \pm 3.2^{(\mathrm{d})}$ & $105.1 \pm 0.4^{(a)}$ & $98.6 \pm 0.2^{(b)}$ & $95.4 \pm 0.2^{(c)}$ \\
\hline Malathion & $102.9 \pm 0.2^{(a)}$ & $113.6 \pm 0.2^{(a)}$ & $109.4 \pm 0.3^{(a)}$ & $98.1 \pm 0.1^{(\mathrm{a})}$ \\
\hline Metalaxyl & $100.3 \pm 0.1^{(a)}$ & $102.8 \pm 0.4^{(a)}$ & $108.1 \pm 0.2^{(a)}$ & $99.3 \pm 0.5^{(a)}$ \\
\hline Metconazole & $56.8 \pm 1.7^{(\mathrm{b})}$ & $101.8 \pm 0.8^{(a)}$ & $109.9 \pm 0.1^{(a)}$ & $101.2 \pm 0.2^{(a)}$ \\
\hline Methidathion & $76.4 \pm 0.7^{(\mathrm{b})}$ & $98.2 \pm 0.4^{(\mathrm{a})}$ & $99.8 \pm 0.5^{(\mathrm{a})}$ & $77.7 \pm 0.2^{(b)}$ \\
\hline Methomyl & $63.9 \pm 7.9^{(a)}$ & $111.1 \pm 0.6^{(a)}$ & $105.6 \pm 0.3^{(a)}$ & $86.0 \pm 0.4^{(a)}$ \\
\hline Metolachlor & $88.6 \pm 0.3^{(a)}$ & $100.2 \pm 0.1^{(\mathrm{a})}$ & $97.7 \pm 0.3^{(a)}$ & $98.4 \pm 0.9^{(a)}$ \\
\hline Metribuzin & $106.3 \pm 0.1^{(a)}$ & $103.9 \pm 0.5^{(\mathrm{a})}$ & $106.0 \pm 0.5^{(a)}$ & $48.7 \pm 0.3^{(b)}$ \\
\hline Metsulfuron-methyl & $72.7 \pm 1.7^{(\mathrm{b})}$ & $46.6 \pm 0.7^{(c)}$ & $72.8 \pm 0.5^{(b)}$ & $122.1 \pm 0.4^{(\mathrm{a})}$ \\
\hline Monocrotophos & $86.4 \pm 0.1^{(\mathrm{a})}$ & $98.4 \pm 0.2^{(a)}$ & $86.3 \pm 0.3^{(a)}$ & $14.7 \pm 7.1^{(\mathrm{b})}$ \\
\hline Nicosulfuron & $43.9 \pm 2.5^{(b)}$ & $19.0 \pm 1.4^{(c)}$ & $42.6 \pm 0.3^{(b)}$ & $100.6 \pm 2.1^{(\mathrm{a})}$ \\
\hline Novaluron & $16.5 \pm 2.5^{(\mathrm{c})}$ & $104.4 \pm 0.2^{(a)}$ & $107.4 \pm 0.3^{(a)}$ & $92.1 \pm 0.6^{(b)}$ \\
\hline Omethoat & $88.0 \pm 0.2^{(b)}$ & $90.6 \pm 0.2^{(a)}$ & $86.4 \pm 0.3^{(b)}$ & $83.5 \pm 0.1^{(\mathrm{b})}$ \\
\hline Oxamyl & $90.3 \pm 0.1^{(\mathrm{a})}$ & $94.7 \pm 0.0^{(\mathrm{a})}$ & $94.4 \pm 0.1^{(\mathrm{a})}$ & $67.8 \pm 0.2^{(\mathrm{b})}$ \\
\hline Picoxystrobin & $76.7 \pm 0.1^{(b)}$ & $94.3 \pm 0.5^{(\mathrm{a})}$ & $92.6 \pm 1.1^{(\mathrm{a})}$ & $79.1 \pm 0.3^{(b)}$ \\
\hline Pirimicarb & $37.1 \pm 2.2^{(c)}$ & $103.6 \pm 1.1^{(a)}$ & $102.3 \pm 0.5^{(a)}$ & $81.2 \pm 0.5^{(b)}$ \\
\hline Pirimiphos-methyl & $44.6 \pm 1.1^{(b)}$ & $99.2 \pm 1.1^{(\mathrm{a})}$ & $99.1 \pm 0.2^{(a)}$ & $91.2 \pm 0.2^{(a)}$ \\
\hline Prochloraz & $34.1 \pm 3.7^{(\mathrm{b})}$ & $106.5 \pm 0.4^{(\mathrm{a})}$ & $111.4 \pm 0.4^{(a)}$ & $103.6 \pm 0.1^{(a)}$ \\
\hline Procymidon & $54.1 \pm 1.0^{(a)}$ & $78.8 \pm 0.7^{(a)}$ & $58.3 \pm 1.7^{(a)}$ & $66.5 \pm 0.7^{(a)}$ \\
\hline Profenofos & $31.7 \pm 2.6^{(\mathrm{b})}$ & $95.9 \pm 0.7^{(a)}$ & $97.2 \pm 0.2^{(a)}$ & $89.1 \pm 0.4^{(a)}$ \\
\hline Promecarb & $106.6 \pm 0.4^{(a)}$ & $107.8 \pm 0.1^{(\mathrm{a})}$ & $102.6 \pm 0.2^{(a)}$ & $95.1 \pm 0.1^{(a)}$ \\
\hline Propamocarb & $75.9 \pm 0.0^{(\mathrm{a})}$ & $49.3 \pm 0.7^{(c)}$ & $57.9 \pm 0.2^{(b)}$ & $73.4 \pm 0.1^{(\mathrm{a})}$ \\
\hline Propargit & $70.1 \pm 1.4^{(\mathrm{ab})}$ & $98.7 \pm 0.4^{(\mathrm{a})}$ & $99.4 \pm 0.7^{(\mathrm{a})}$ & $64.2 \pm 0.2^{(a b)}$ \\
\hline Pyraclostrobin & $5.2 \pm 0.9^{(c)}$ & $111.8 \pm 0.0^{(a)}$ & $106.7 \pm 0.2^{(a)}$ & $100.3 \pm 0.3^{(b)}$ \\
\hline Pyridaben & $53.1 \pm 2.0^{(\mathrm{b})}$ & $100.0 \pm 0.8^{(a)}$ & $102.6 \pm 0.3^{(a)}$ & $89.9 \pm 1.3^{(a)}$ \\
\hline Pyriproxyfen & $36.9 \pm 2.6^{(\mathrm{b})}$ & $98.1 \pm 0.5^{(\mathrm{a})}$ & $97.9 \pm 0.3^{(a)}$ & $92.3 \pm 0.1^{(\mathrm{a})}$ \\
\hline Rotenone & $46.7 \pm 2.7^{(\mathrm{b})}$ & $101.2 \pm 0.5^{(a)}$ & $100.1 \pm 0.5^{(a)}$ & $96.5 \pm 0.1^{(\mathrm{a})}$ \\
\hline Spinosyn A & $12.5 \pm 2.6^{(\mathrm{d})}$ & $98.3 \pm 0.3^{(b)}$ & $109.2 \pm 0.7^{(a)}$ & $87.0 \pm 0.5^{(c)}$ \\
\hline Spinosyn D & $9.3 \pm 3.2^{(c)}$ & $92.0 \pm 0.1^{(b)}$ & $101.6 \pm 0.4^{(a)}$ & $90.5 \pm 0.3^{(b)}$ \\
\hline Tebuconazole & $59.7 \pm 1.6^{(\mathrm{b})}$ & $99.5 \pm 0.7^{(a)}$ & $114.4 \pm 0.6^{(a)}$ & $111.4 \pm 0.4^{(\mathrm{a})}$ \\
\hline Tebufenozid & $100.9 \pm 0.5^{(b)}$ & $112.0 \pm 0.4^{(a)}$ & $120.4 \pm 1.5^{(\mathrm{a})}$ & $98.6 \pm 0.4^{(b)}$ \\
\hline Temephos & $25.4 \pm 3.0^{(c)}$ & $105.9 \pm 0.4^{(a)}$ & $100.7 \pm 0.2^{(\mathrm{ab})}$ & $98.0 \pm 0.6^{(b)}$ \\
\hline Tetraconazole & $92.5 \pm 0.6^{(c)}$ & $108.2 \pm 1.0^{(\mathrm{a})}$ & $102.4 \pm 0.5^{(\mathrm{ab})}$ & $93.4 \pm 0.6^{(b c)}$ \\
\hline
\end{tabular}


Table 2 Percentage recoveries ( \pm SD) of 96 pesticides subjected to either QuEChERS clean-up methods or no clean-up (Continued)

\begin{tabular}{|c|c|c|c|c|}
\hline Thiabendazol & $17.2 \pm 1.6^{(\mathrm{d})}$ & $82.2 \pm 0.4^{(a)}$ & $77.3 \pm 0.1^{(b)}$ & $57.9 \pm 0.2^{(c)}$ \\
\hline Thiacloprid & $85.3 \pm 1.2^{(a)}$ & $99.5 \pm 0.6^{(a)}$ & $94.7 \pm 0.0^{(a)}$ & $61.0 \pm 0.3^{(b)}$ \\
\hline Thiamethoxam & $95.6 \pm 0.6^{(a)}$ & $101.2 \pm 0.1^{(a)}$ & $99.7 \pm 0.2^{(\mathrm{a})}$ & $59.7 \pm 0.1^{(b)}$ \\
\hline Thiodicarb & $43.3 \pm 2.4^{(\mathrm{c})}$ & $101.4 \pm 0.6^{(a)}$ & $103.5 \pm 0.5^{(a)}$ & $76.8 \pm 0.3^{(b)}$ \\
\hline Triadimenol & $92.8 \pm 0.1^{(\mathrm{b})}$ & $108.6 \pm 1.0^{(\mathrm{a})}$ & $111.3 \pm 0.03^{(\mathrm{a})}$ & $97.9 \pm 0.6^{(a)}$ \\
\hline Triadimefon & $117.7 \pm 0.3^{(\mathrm{a})}$ & $107.4 \pm 0.8^{(a)}$ & $115.7 \pm 0.1^{(\mathrm{a})}$ & $110.6 \pm 0.7^{(a)}$ \\
\hline Tribenuron-methyl & $64.2 \pm 1.7^{(b)}$ & $73.3 \pm 0.1^{(\mathrm{a})}$ & $81.5 \pm 0.1^{(a)}$ & $63.4 \pm 0.4^{(b)}$ \\
\hline Trifloxystrobin & $60.1 \pm 1.7^{(\mathrm{b})}$ & $101.2 \pm 0.2^{(\mathrm{a})}$ & $103.8 \pm 0.1^{(\mathrm{a})}$ & $93.7 \pm 0.1^{(\mathrm{a})}$ \\
\hline
\end{tabular}

*For each pesticide, mean recoveries with the same letter are not significantly different

MRM methods of targeted analytes as shown in Fig. 3. This figure illustrates an example of MRM chromatogram of the 96 pesticides targeted in this study that were extracted from spiked honey after PSA clean up. From this chromatogram, each colored peak represent a unique pesticide identified based on the MRM transition ions. A detailed summary indicating the identity of each peak shown in Fig. 3 and their corresponding retention times along with their molecular masses are provided in Table 1.

\section{Validation of the selected method}

The developed method was validated following the guidelines provided in the Guidance document on analytical quality control and validation procedures for pesticide residues analysis in food and feed (SANCO/12571/2013). To meet these guidelines, the method was validated in terms of recovery, linearity, LOQ, matrix effects, intra-day and inter-day precision. The mean recovery values used in this study were within the range of 70-120\%, with an associated repeatability, RSD $<20 \%$, for all compounds within the scope of the method. Matrix-matched calibration standards were used to calculate recoveries as this helped in compensating for any matrix effects arising from matrix interferences or co-extractives that can change the ionization efficiency of an analyte causing signal suppression or enhancement leading to poor recoveries. This could have an adverse effect on the quality of the data and can erroneously result in false positive or negative results. It is therefore imperative for any LC-MS/MS method to give acceptable quantitative results; matrix effects must be considered (Ferrer et al. 2011; Kittlaus et al. 2011).

Table 3 shows the list of pesticides validated and demonstrates the summarized recovery results along with the linearity of the validated analytes. This table illustrates recoveries obtained at LOR using PSA and no clean-up approach. Percent recovery values for these analytes were calculated using matrix-matched calibration curves. The LOR for the method was determined as the lowest spike level of the validation meeting these method performance acceptability criteria. Although the LOD and LOR varied depending on the pesticides in question, most compounds could be detected at 0.1 and quantified below $1 \mathrm{ng} / \mathrm{g}$. Overall, the LOD and the LOR was set at 0.5 and $1 \mathrm{ng} / \mathrm{g}$, respectively. From this study, approximately $10 \%$ of the studied compounds had poor recoveries from either method but there was tremendous improvement on recoveries when both methods were combined. In this case, all pesticides, except for two (fluquinconazole $-68 \%$ and propamocarb - $63 \%$ ) had good recoveries which were well within the recommended limits provided in SANCO/ $12575 / 2013$ document. It is worth noting that pesticides with good recoveries had good reproducibility (RSD $<20 \%$ ) whereas those with poor recoveries were characterized by poor reproducibility. As a result, during

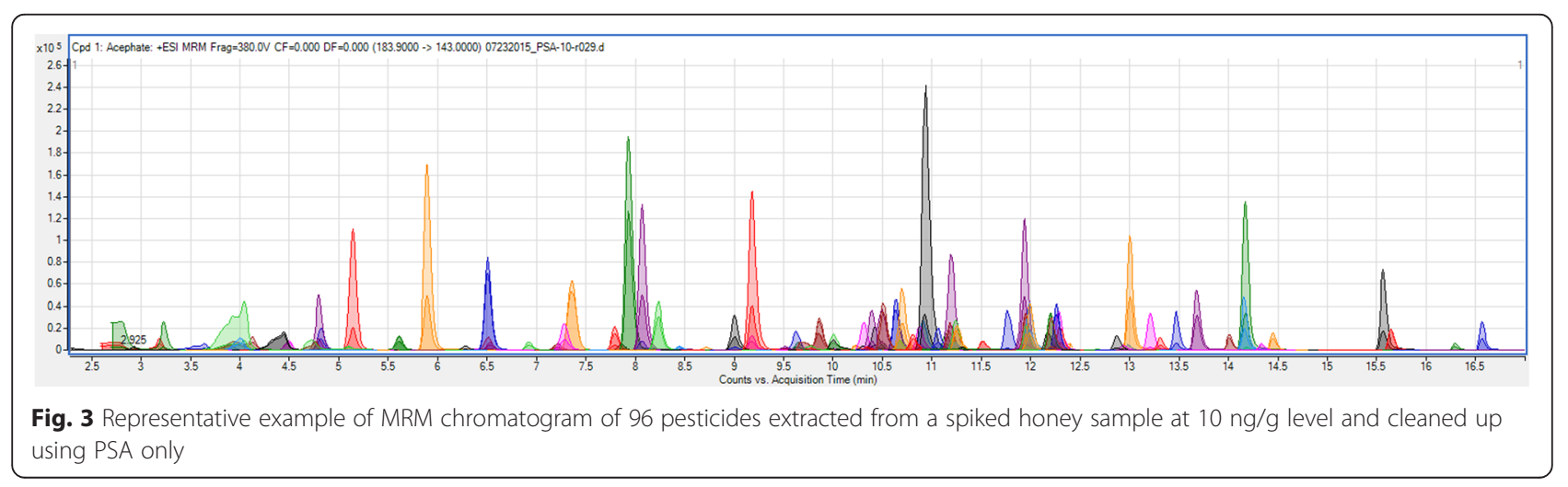


Table 3 Extraction efficiencies of validated pesticides spiked at LOR, precision in terms of RSD $(n=5)$ and coefficients of determination for the investigated pesticides

\begin{tabular}{|c|c|c|c|c|c|c|c|c|c|c|c|}
\hline \multirow{2}{*}{\multicolumn{5}{|c|}{ \% recovery at LOR $(1 \mathrm{ng} / \mathrm{g})$}} & & \\
\hline & & & & & & \multirow{3}{*}{$\begin{array}{l}\text { Flazasulfuron } \\
\text { Fludioxonil } \\
\text { Flufenacet }\end{array}$} & \multirow{3}{*}{$\begin{array}{l}87.1 \\
75.6 \\
82.2\end{array}$} & \multirow{3}{*}{$\begin{array}{r}3.7 \\
5.3 \\
14.1\end{array}$} & \multirow{3}{*}{$\begin{array}{l}25.7 \\
62.2 \\
79.1\end{array}$} & \multirow{3}{*}{$\begin{array}{r}62.6 \\
1.8 \\
17.0\end{array}$} & \multirow{3}{*}{$\begin{array}{l}0.9995 \\
0.9981 \\
0.9926\end{array}$} \\
\hline \multirow[b]{2}{*}{ Compound name } & \multicolumn{2}{|l|}{ No clean-up } & \multicolumn{2}{|l|}{ PSA } & \multirow[b]{2}{*}{$\mathrm{R} \wedge 2$} & & & & & & \\
\hline & $\%$ recovery & $\begin{array}{l}\% \text { RSD, } \\
n=5\end{array}$ & \% recovery & $\begin{array}{l}\% \text { RSD, } \\
n=5\end{array}$ & & & & & & & \\
\hline Acephate & 70.1 & 2.6 & 97.4 & 4.7 & 0.9989 & Fluquinconazole & 68.3 & 6.9 & 68.3 & 1.7 & 0.9981 \\
\hline Acetamiprid & 82.6 & 3.2 & 115.0 & 3.9 & 0.9982 & Flusilazole & 79.5 & 4.7 & 90.9 & 6.1 & 0.9991 \\
\hline Aldicarb fragment & 73.0 & 0.9 & 102.8 & 1.5 & 0.9476 & Flutriafol & 93.5 & 4.9 & 93.9 & 1.8 & 0.9999 \\
\hline Amidosulfuron & 87.1 & 1.2 & 54.2 & 24.3 & 0.9990 & Fosthiazate & 73.2 & 4.1 & 109.4 & 1.6 & 0.9998 \\
\hline Azoxystrobin & 84.5 & 4.5 & 90.8 & 4.9 & 0.9986 & Hexaconazole & 94.6 & 0.3 & 98.8 & 0.9 & 1.0000 \\
\hline Benalaxyl & 87.6 & 3.3 & 87.5 & 1.5 & 0.9989 & Hexythiazox & 89.7 & 2.7 & 98.2 & 0.8 & 0.9997 \\
\hline Bifenazate & 89.6 & 2.4 & 96.3 & 4.3 & 0.9919 & Imidacloprid & 69.3 & 6.0 & 101.4 & 3.4 & 0.9996 \\
\hline Bifenthrin & 91.3 & 6.5 & 99.0 & 5.0 & 0.9996 & Indoxacarb & 93.2 & 1.1 & 96.8 & 1.9 & 0.9998 \\
\hline Bitertanol & 85.7 & 0.4 & 89.9 & 0.1 & 0.9982 & Ipconazole & 89.8 & 2.4 & 95.7 & 4.9 & 0.9996 \\
\hline Bosclid (Nicobifen) & 81.7 & 0.7 & 88.2 & 1.5 & 0.9977 & Iprovalicarb & 90.7 & 13.6 & 100.6 & 9.9 & 0.9988 \\
\hline Bromuconazole & 117.3 & 11.5 & 102.3 & 9.3 & 0.9991 & Isoxaflutole & 91.4 & 0.5 & 76.0 & 5.7 & 0.9951 \\
\hline Bupirimate & 87.6 & 1.7 & 95.6 & 4.1 & 0.9998 & Kresoxim-methyl & 108.8 & 10.7 & 113.5 & 13.2 & 0.9962 \\
\hline Buprofezin & 81.9 & 9.0 & 86.1 & 4.3 & 0.9985 & Linuron & 76.3 & 7.1 & 74.0 & 3.0 & 0.9958 \\
\hline Carbaryl & 83.8 & 1.8 & 118.3 & 1.3 & 0.9980 & Lufenuron & 91.9 & 15.8 & 90.2 & 9.0 & 0.9990 \\
\hline Carbofuran & 67.8 & 3.1 & 115.8 & 5.1 & 0.9987 & Malathion & 78.8 & 8.3 & 86.3 & 1.0 & 0.9972 \\
\hline Chlorfenvinphos & 95.0 & 5.7 & 103.0 & 1.1 & 1.0000 & Metalaxyl & 83.1 & 1.3 & 91.4 & 0.7 & 0.9995 \\
\hline Chlorpyriphos & 93.1 & 6.2 & 99.3 & 1.8 & 0.9996 & Metconazole & 78.2 & 11.0 & 81.0 & 8.2 & 0.9984 \\
\hline \multirow{2}{*}{$\begin{array}{l}\text { Chlorpyriphos- } \\
\text { methyl }\end{array}$} & \multirow[t]{2}{*}{82.9} & \multirow[t]{2}{*}{16.9} & \multirow[t]{2}{*}{92.7} & \multirow[t]{2}{*}{8.6} & 0.9990 & Methidathion & 72.7 & 8.2 & 89.5 & 2.6 & 0.9993 \\
\hline & & & & & & Methomyl & 96.1 & 0.4 & 114.6 & 3.5 & 0.9996 \\
\hline Clofentezin & 93.8 & 1.8 & 94.0 & 1.9 & 0.9996 & Metolachlor & 111.6 & 1.1 & 115.3 & 3.7 & 0.9984 \\
\hline Coumaphos & 70.3 & 4.7 & 75.9 & 3.3 & 0.9935 & Metribuzin & 66.3 & 14.3 & 115.4 & 2.8 & 0.9976 \\
\hline Cyazofamid & 108.4 & 9.4 & 113.6 & 1.6 & 0.9992 & Metsulfuron-methyl & 121.6 & 0.5 & 53.5 & 28.1 & 0.9994 \\
\hline Cymiazol & 86.6 & 0.5 & 109.6 & 5.9 & 0.9987 & Monocrotophos & 77.9 & 0.4 & 112.5 & 2.7 & 0.9968 \\
\hline Cyproconazole & 84.8 & 0.9 & 89.2 & 4.1 & 0.9976 & Nicosulfuron & 90.1 & 2.1 & 10.5 & 39.9 & 0.9994 \\
\hline Cyprodinil & 93.7 & 1.0 & 96.7 & 1.6 & 0.9995 & Novaluron & 94.9 & 0.2 & 95.5 & 0.6 & 0.9999 \\
\hline DEET & 68.5 & 3.3 & 96.1 & 4.0 & 0.9999 & Omethoate & 78.3 & 5.9 & 81.3 & 15.8 & 0.9995 \\
\hline Diazinon & 87.6 & 3.6 & 100.4 & 0.5 & 0.9999 & Oxamyl & 72.9 & 15.3 & 112.4 & 2.5 & 0.9995 \\
\hline Dichlorvos & 72.8 & 15.4 & 101.4 & 10.9 & 0.9998 & Picoxystrobin & 93.1 & 2.6 & 101.4 & 9.4 & 0.9992 \\
\hline Diflubenzuron & 95.6 & 0.4 & 99.5 & 3.9 & 0.9999 & Pirimicarb & 87.0 & 2.5 & 113.1 & 0.4 & 0.9992 \\
\hline Dimethoate & 67.5 & 2.9 & 108.7 & 0.3 & 0.9997 & Pirimiphos-methyl & 99.3 & 1.0 & 112.2 & 4.4 & 0.9999 \\
\hline Diuron & 55.9 & 0.7 & 98.4 & 4.7 & 0.9979 & Prochloraz & 79.9 & 0.4 & 80.1 & 1.2 & 0.9978 \\
\hline Epoxyconazol & 100.4 & 8.4 & 100.7 & 0.4 & 1.0000 & Procymidon & 79.9 & 6.0 & 111.7 & 10.9 & 0.9956 \\
\hline Ethion & 74.9 & 7.1 & 82.8 & 8.9 & 0.9986 & Profenofos & 98.5 & 3.7 & 109.2 & 11.2 & 0.9994 \\
\hline Ethoprophos & 91.6 & 3.7 & 103.8 & 11.8 & 0.9998 & Promecarb & 85.1 & 1.3 & 87.7 & 0.2 & 0.9993 \\
\hline Etofenprox & 95.2 & 0.8 & 101.3 & 2.8 & 1.0000 & Propamocarb & 62.8 & 0.0 & 29.7 & 83.9 & 0.9996 \\
\hline Fenamiphos & 100.2 & 8.9 & 103.8 & 2.0 & 0.9991 & Propargit & 25.2 & 52.7 & 76.5 & 19.8 & 0.9983 \\
\hline Fenazaquin & 95.1 & 2.3 & 99.5 & 5.2 & 0.9999 & Pyraclostrobin & 80.0 & 10.4 & 88.1 & 5.6 & 0.9972 \\
\hline Fenbuconazole & 94.9 & 1.9 & 101.3 & 0.4 & 1.0000 & Pyridaben & 84.3 & 1.5 & 87.2 & 1.2 & 0.9998 \\
\hline Fenobucarb & 83.0 & 5.1 & 96.7 & 0.3 & 0.9999 & Pyriproxyfen & 92.7 & 4.4 & 100.4 & 1.0 & 1.0000 \\
\hline Fipronil & 99.7 & 2.6 & 98.3 & 13.7 & 0.9982 & Rotenone & 97.7 & 1.9 & 113.0 & 1.7 & 0.9985 \\
\hline
\end{tabular}

Table 3 Extraction efficiencies of validated pesticides spiked at LOR, precision in terms of RSD $(n=5)$ and coefficients of determination for the investigated pesticides (Continued) 
Table 3 Extraction efficiencies of validated pesticides spiked at $\mathrm{LOR}$, precision in terms of RSD $(n=5)$ and coefficients of determination for the investigated pesticides (Continued)

\begin{tabular}{llllll}
\hline Spinosyn A & 92.0 & 0.8 & 95.9 & 0.8 & 0.9999 \\
Spinosyn D & 85.1 & 0.1 & 92.3 & 2.0 & 0.9997 \\
Tebuconazole & 92.1 & 4.0 & 97.1 & 0.1 & 0.9993 \\
Tebufenozid & 75.3 & 0.5 & 87.7 & 9.5 & 0.9958 \\
Temephos & 94.4 & 1.6 & 95.5 & 2.6 & 0.9999 \\
Tetraconazole & 83.1 & 6.1 & 108.6 & 3.1 & 0.9997 \\
Thiabendazol & 91.3 & 2.7 & 110.9 & 1.9 & 0.9946 \\
Thiacloprid & 67.7 & 0.4 & 108.2 & 1.8 & 0.9995 \\
Thiamethoxam & 55.4 & 5.5 & 100.0 & 1.6 & 1.0000 \\
Thiodicarb & 78.3 & 1.0 & 102.4 & 3.5 & 0.9996 \\
Triadimenol & 73.7 & 8.5 & 69.8 & 13.5 & 0.9903 \\
Triadimefon & 85.6 & 8.9 & 83.8 & 2.2 & 0.9948 \\
Tribenuron-methyl & 71.8 & 4.9 & 65.7 & 13.4 & 0.9997 \\
Trifloxystrobin & 94.7 & 3.1 & 100.6 & 0.6 & 0.9999 \\
\hline
\end{tabular}

recovery studies, blank matrix was fortified at 10 times the LOR since it gave the best reproducibility for all studied compounds. The method linearity was evaluated by assessing the signal responses of the targeted analytes from matrix-matched calibration solutions prepared in blank extracts at seven concentration levels. The developed method was proven satisfactory with linear chromatographic response for the tested pesticides, ranging from 0.1 to $100 \mathrm{ng} \mathrm{g}^{-1}$. Majority of the correlation coefficients $\left(R^{2}\right)$ was higher or equal to 0.995 , see Table 3.

\section{Application of the method to real samples}

As a natural product manufactured by bees, honey is considered to be free from any extraneous material. However, chemical residues have been reported in honey by several investigators. The presence of these residues in honey has prompted the need for setting up monitoring programs to determine the proper assessment of human exposure to pesticides (Choudhary and Sharma 2008). Unfortunately, there is no homogeneity on MRLs as different national regulations have established their own maximum concentrations of pesticide residues permitted in honey. In the absence of MRLs set for honey in the two African countries studied, the European Union set MRLs were employed and where no MRL existed, it was presumed at 10 which is the default MRL for pesticides with no specific value set as recommended in Regulation(EC)No 396/2005.

So far, there is little information that is currently available on chemical residues present in honey or hive products from most African countries (Muli et al. 2014; Eissa et al. 2014). Previous studies have shown that whereas in North America honey bees are exposed to at least 7 pesticides per food visit, this is not the case in Africa (Mullin et al. 2010). Results from a recent study carried out in Kenya detected less than four pesticides for the whole study duration at very minimal concentrations in honey bees and their hive products (Muli et al. 2014). In the current study, a preliminary analysis of pesticide residues in 28 honey samples obtained from local farmers' markets and supermarkets from various regions in Kenya and Ethiopia during the period of November 2014 to July 2015 revealed the presence of 17 pesticide residues out of the 96 pesticides investigated. The concentrations for each detected pesticide were compared with the set MRL values. Table 4 indicates the summarized results obtained from the two countries. Our preliminary results show that, with the exception of malathion, an organophosphate that has multiple uses in Africa, no other pesticide was detected at a level higher than the set MRL levels. For most pesticides, the levels obtained were about 10-fold lower than the set MRL levels, with concentration levels at $<100 \mathrm{ng} / \mathrm{g}$. However, the maximum concentration detected for malathion was $0.092 \mathrm{mg} / \mathrm{kg}$, a level that far exceeds its acceptable MRL of $0.05 \mathrm{mg} /$ $\mathrm{kg}$. Although this compound is quickly metabolized from the body and is known to be non-persistent in the environment, exposure to the levels detected $(0.092 \mathrm{mg} / \mathrm{kg})$ in this study over a long period could result in adverse health effects to both humans and honey bees. Thus, further investigation is required to determine its cumulative effects and whether there are any potential synergistic effects when other contaminants are present. Malathion is also considered to be highly toxic to honey bees with $\mathrm{LD}_{50}$ of $0.16 \mu \mathrm{g} /$ bee (Allison 2011). It is worth noting that data from the present study does not reflect seasonality of pesticide present in honey samples obtained from the two countries. This would require in-depth systematic studies using large samples obtained directly from specific beekeeping sites over different seasons in the two countries. Follow up studies are underway to investigate how seasonality affects residues present in honey from various African countries.

\section{Conclusion}

A highly efficient approach for determining pesticide residues in honey with good recoveries was developed. This approach involved using a modified QuEChERS method along with or without any clean-up. The viability of this approach was demonstrated by using 96 pesticides. About $98 \%$ of these pesticides investigated had recoveries that are well within the acceptable limits of $70-120 \%$. The methods were linear $(>0.995)$ over the range tested $(0.1-100 \mathrm{ng} / \mathrm{g})$ with LOR for 
Table 4 Detected pesticide residues in honey obtained from Kenya and Ethiopia

\begin{tabular}{|c|c|c|c|c|c|c|c|c|c|c|c|c|c|c|c|c|c|}
\hline \multirow[b]{2}{*}{ SamplelD } & \multicolumn{17}{|c|}{ Identified pesticide residues } \\
\hline & ACTM & AF & CF & CAR & $\mathrm{CHP}$ & Cy & DEET & DDVP & DM & BPMC & HEX & Mal & Met & Metri & Rot & TBN & THIA \\
\hline \multicolumn{18}{|l|}{ Kenya } \\
\hline Taita & $<\mathrm{LOQ}$ & $\mathrm{N} / \mathrm{D}$ & N/D & N/D & $<\mathrm{LOQ}$ & $<\mathrm{LOQ}$ & 0.708 & N/D & $\mathrm{N} / \mathrm{D}$ & N/D & $<\mathrm{LOQ}$ & 56.9 & $N / D$ & 49.4 & N/D & $<\mathrm{LOQ}$ & $<\mathrm{LOQ}$ \\
\hline VapA & $<\mathrm{LOQ}$ & 1.37 & $<\mathrm{LOQ}$ & N/D & $<\mathrm{LOQ}$ & $\mathrm{N} / \mathrm{D}$ & N/D & $N / D$ & N/D & N/D & $\mathrm{N} / \mathrm{D}$ & 92.3 & 1.81 & $N / D$ & N/D & N/D & $<\mathrm{LOQ}$ \\
\hline Cab & $<\mathrm{LOQ}$ & $\mathrm{N} / \mathrm{D}$ & N/D & N/D & $<\mathrm{LOQ}$ & 1.59 & $<\mathrm{LOQ}$ & N/D & N/D & $<\mathrm{LOQ}$ & $<\mathrm{LOQ}$ & N/D & 1.95 & 14.1 & N/D & N/D & N/D \\
\hline Nak & N/D & $<\mathrm{LOQ}$ & N/D & $<\mathrm{LOQ}$ & N/D & N/D & $<\mathrm{LOQ}$ & N/D & $\mathrm{N} / \mathrm{D}$ & $\mathrm{N} / \mathrm{D}$ & N/D & N/D & $\mathrm{N} / \mathrm{D}$ & N/D & N/D & $\mathrm{N} / \mathrm{D}$ & N/D \\
\hline Ken & $<\mathrm{LOQ}$ & $<\mathrm{LOQ}$ & N/D & N/D & $\mathrm{N} / \mathrm{D}$ & N/D & $<\mathrm{LOQ}$ & N/D & N/D & N/D & N/D & N/D & $N / D$ & N/D & N/D & N/D & N/D \\
\hline Mwi & N/D & N/D & N/D & 1.26 & $\mathrm{~N} / \mathrm{D}$ & N/D & 1.01 & $\mathrm{~N} / \mathrm{D}$ & $<\mathrm{LOQ}$ & N/D & $\mathrm{N} / \mathrm{D}$ & N/D & $\mathrm{N} / \mathrm{D}$ & $\mathrm{N} / \mathrm{D}$ & N/D & N/D & N/D \\
\hline Kak & $<\mathrm{L} / \mathrm{OQ}$ & $<\mathrm{LOQ}$ & N/D & N/D & $\mathrm{N} / \mathrm{D}$ & $<\mathrm{LOQ}$ & $<\mathrm{LOQ}$ & N/D & $\mathrm{N} / \mathrm{D}$ & N/D & $\mathrm{N} / \mathrm{D}$ & N/D & $N / D$ & $<\mathrm{LOQ}$ & N/D & N/D & $\mathrm{N} / \mathrm{D}$ \\
\hline $\mathrm{ML}$ & ND & $\mathrm{N} / \mathrm{D}$ & N/D & $<\mathrm{LOQ}$ & $\mathrm{N} / \mathrm{D}$ & N/D & $<\mathrm{LOQ}$ & N/D & N/D & N/D & N/D & N/D & $N / D$ & 34.0 & N/D & N/D & N/D \\
\hline$H R$ & $<\mathrm{LOQ}$ & $\mathrm{N} / \mathrm{D}$ & $\mathrm{N} / \mathrm{D}$ & 2.87 & $\mathrm{~N} / \mathrm{D}$ & $<\mathrm{LOQ}$ & $<\mathrm{LOQ}$ & N/D & N/D & N/D & $\mathrm{N} / \mathrm{D}$ & N/D & $\mathrm{N} / \mathrm{D}$ & 70.4 & N/D & N/D & N/D \\
\hline Gedi & $\mathrm{N} / \mathrm{D}$ & $\mathrm{N} / \mathrm{D}$ & N/D & N/D & $\mathrm{N} / \mathrm{D}$ & N/D & N/D & $\mathrm{N} / \mathrm{D}$ & N/D & N/D & N/D & N/D & $N / D$ & $\mathrm{~N} / \mathrm{D}$ & N/D & N/D & N/D \\
\hline$K-B$ & N/D & N/D & N/D & $<\mathrm{LOQ}$ & N/D & N/D & 1.37 & 2.58 & N/D & N/D & N/D & N/D & $\mathrm{N} / \mathrm{D}$ & N/D & N/D & N/D & $\mathrm{N} / \mathrm{D}$ \\
\hline K-M & $\mathrm{N} / \mathrm{D}$ & N/D & N/D & N/D & N/D & N/D & $<\mathrm{LOQ}$ & $<\mathrm{LOQ}$ & $<\mathrm{LOQ}$ & N/D & $\mathrm{N} / \mathrm{D}$ & N/D & $\mathrm{N} / \mathrm{D}$ & $<\mathrm{LOQ}$ & N/D & N/D & $\mathrm{N} / \mathrm{D}$ \\
\hline K-N & $<L O Q$ & N/D & N/D & $<\mathrm{LOQ}$ & N/D & N/D & $<L O Q$ & N/D & N/D & N/D & N/D & N/D & $\mathrm{N} / \mathrm{D}$ & N/D & N/D & N/D & $\mathrm{N} / \mathrm{D}$ \\
\hline VapB & $<\mathrm{LOQ}$ & $<\mathrm{LOQ}$ & N/D & N/D & $<\mathrm{LOQ}$ & N/D & 1.09 & $\mathrm{~N} / \mathrm{D}$ & N/D & N/D & 1.66 & 76.7 & 5.29 & N/D & N/D & $<\mathrm{LOQ}$ & $<\mathrm{LOQ}$ \\
\hline \multicolumn{18}{|l|}{ Ethiopia } \\
\hline $\mathrm{MB}$ & $\mathrm{N} / \mathrm{D}$ & $\mathrm{N} / \mathrm{D}$ & N/D & $\mathrm{N} / \mathrm{D}$ & $\mathrm{N} / \mathrm{D}$ & N/D & $\mathrm{N} / \mathrm{D}$ & $\mathrm{N} / \mathrm{D}$ & N/D & N/D & $\mathrm{N} / \mathrm{D}$ & N/D & $N / D$ & 9.52 & N/D & N/D & $\mathrm{N} / \mathrm{D}$ \\
\hline Tol & $<\mathrm{LOQ}$ & $\mathrm{N} / \mathrm{D}$ & N/D & $\mathrm{N} / \mathrm{D}$ & $<\mathrm{LOQ}$ & $<\mathrm{LOQ}$ & $<\mathrm{LOQ}$ & N/D & N/D & $<\mathrm{LOQ}$ & $\mathrm{N} / \mathrm{D}$ & 60.5 & 4.77 & 11.2 & N/D & $<\mathrm{LOQ}$ & $\mathrm{N} / \mathrm{D}$ \\
\hline Tig & $<\mathrm{LOQ}$ & $<\mathrm{LOQ}$ & N/D & N/D & $<\mathrm{LOQ}$ & $<\mathrm{LOQ}$ & $<\mathrm{LOQ}$ & N/D & N/D & N/D & $<\mathrm{LOQ}$ & 15.3 & $N / D$ & N/D & N/D & N/D & $\mathrm{N} / \mathrm{D}$ \\
\hline SapV & $<\mathrm{LOQ}$ & $<\mathrm{LOQ}$ & $<\mathrm{LOQ}$ & N/D & $<\mathrm{LOQ}$ & $<\mathrm{LOQ}$ & $<\mathrm{LOQ}$ & N/D & N/D & $<\mathrm{LOQ}$ & $<\mathrm{LOQ}$ & 45.1 & 1.25 & 14.2 & N/D & $<\mathrm{LOQ}$ & $<\mathrm{LOQ}$ \\
\hline$E-1$ & N/D & N/D & N/D & N/D & N/D & N/D & $\mathrm{N} / \mathrm{D}$ & $\mathrm{N} / \mathrm{D}$ & N/D & N/D & N/D & N/D & N/D & 2.60 & N/D & N/D & $\mathrm{N} / \mathrm{D}$ \\
\hline$E-2$ & $\mathrm{~N} / \mathrm{D}$ & $\mathrm{N} / \mathrm{D}$ & N/D & N/D & $\mathrm{N} / \mathrm{D}$ & N/D & N/D & 1.16 & $<\mathrm{LOQ}$ & N/D & N/D & N/D & $\mathrm{N} / \mathrm{D}$ & 44.2 & N/D & N/D & $\mathrm{N} / \mathrm{D}$ \\
\hline E-3 & $<\mathrm{LOQ}$ & $\mathrm{N} / \mathrm{D}$ & N/D & N/D & $\mathrm{N} / \mathrm{D}$ & N/D & $\mathrm{N} / \mathrm{D}$ & $\mathrm{N} / \mathrm{D}$ & N/D & N/D & N/D & N/D & N/D & $\mathrm{N} / \mathrm{D}$ & N/D & N/D & $\mathrm{N} / \mathrm{D}$ \\
\hline$E-4$ & ND & N/D & N/D & N/D & N/D & N/D & N/D & N/D & N/D & N/D & N/D & N/D & $\mathrm{N} / \mathrm{D}$ & 7.95 & 6.99 & N/D & $\mathrm{N} / \mathrm{D}$ \\
\hline$E-5$ & ND & $\mathrm{N} / \mathrm{D}$ & N/D & N/D & $\mathrm{N} / \mathrm{D}$ & N/D & N/D & $\mathrm{N} / \mathrm{D}$ & N/D & N/D & $\mathrm{N} / \mathrm{D}$ & N/D & $N / D$ & $\mathrm{~N} / \mathrm{D}$ & N/D & N/D & N/D \\
\hline E-M1 & $<\mathrm{LOQ}$ & $<\mathrm{LOQ}$ & N/D & $<\mathrm{LOQ}$ & N/D & N/D & N/D & $\mathrm{N} / \mathrm{D}$ & N/D & N/D & $\mathrm{N} / \mathrm{D}$ & N/D & $\mathrm{N} / \mathrm{D}$ & N/D & N/D & N/D & $\mathrm{N} / \mathrm{D}$ \\
\hline $\mathrm{E}-\mathrm{H}$ & $<\mathrm{LOQ}$ & $\mathrm{N} / \mathrm{D}$ & N/D & $<\mathrm{LOQ}$ & N/D & N/D & N/D & $\mathrm{N} / \mathrm{D}$ & N/D & N/D & $\mathrm{N} / \mathrm{D}$ & N/D & $\mathrm{N} / \mathrm{D}$ & N/D & N/D & N/D & N/D \\
\hline Tol2 & N/D & $\mathrm{N} / \mathrm{D}$ & 1.10 & N/D & $\mathrm{N} / \mathrm{D}$ & N/D & N/D & 3.46 & $<\mathrm{LOQ}$ & N/D & N/D & N/D & $\mathrm{N} / \mathrm{D}$ & N/D & N/D & N/D & $\mathrm{N} / \mathrm{D}$ \\
\hline Tig2 & $\mathrm{N} / \mathrm{D}$ & N/D & N/D & N/D & N/D & N/D & 4.98 & N/D & N/D & N/D & $\mathrm{N} / \mathrm{D}$ & N/D & N/D & N/D & N/D & N/D & $\mathrm{N} / \mathrm{D}$ \\
\hline Sap S2 & $<\mathrm{LOQ}$ & N/D & $<\mathrm{LOQ}$ & $\mathrm{N} / \mathrm{D}$ & $<\mathrm{LOQ}$ & 10.5 & $<\mathrm{LOQ}$ & N/D & N/D & $<\mathrm{LOQ}$ & $<\mathrm{LOQ}$ & 22.3 & $N / D$ & 21.0 & N/D & N/D & $\mathrm{N} / \mathrm{D}$ \\
\hline MRL & 50 & 10 & 10 & 50 & ${ }^{*} 10$ & ${ }^{*} 10$ & ${ }^{*} 10$ & ${ }^{*} 10$ & ${ }^{*} 10$ & ${ }^{*} 10$ & ${ }^{*} 10$ & 50 & 50 & 100 & 10 & 50 & 10 \\
\hline
\end{tabular}

*Set at default MRL value; $N / D$ not detected, $<L O Q$ below the quantification limits

Identified pesticide residues: ACTM Acetamiprid, AF Aldicarb fragment, CF Carbofuran, CHP Chlopyrifos, Cy Cymiazole, DDVP Dichlorvos, DM Dimethoate,

BPMC Fenobucarb, HEX Hexaconazole, Mal Malathion, Met Metalaxyl, Metri Metribuzin, Rot Rotenone, TBN Tebuconazole, THIA Thiomethoxam

most pesticides at $1 \mathrm{ng} / \mathrm{g}$ or ppb. The applicability of the developed methods to real samples was tested by performing a preliminary study of commercial honey from Africa. A total of 17 pesticide residues were detected at levels 10-fold lower than their set MRL values except malathion which was detected at almost 2-fold higher than its set MRL. Overall, these results suggest that honey from these regions maybe safe for both bees and human consumption but further investigation is required to determine the cumulative effect of these pesticides. In-depth follow up studies using this method are underway to verify this observation in honey samples collected from different agro-ecological regions from various African countries.

\section{Acknowledgements}

The authors would like to thank icipe management for their support, Daisy Salifu of icipe, for her support in statistical analysis, colleagues from African Reference Laboratory Bee Health (ARLBH) at icipe for their support, Beatrice Njuguna of ARLBH for providing the honeybee samples for analysis. This work has been supported financially by the European Union grant number DCI-FOOD-2013/313-659. 


\section{Authors' contributions}

$J$ designed the study and the experimental setting, performed the analytical work and wrote the manuscript. BT contributed in experimental design and critically revised the manuscript. SK edited and proofread the manuscript. All authors read and approved the final manuscript.

\section{Competing interests}

The authors declare that they have no competing interests.

Received: 30 March 2016 Accepted: 18 August 2016

Published online: 02 September 2016

\section{References}

Allison C. Malathion.toxipedia. 2011. http://www.toxipedia.org/display/toxipedia/ Malathion. Accessed 13 July 2015

Anastassiades M, Lehotay SJ, Stajnbaher D, Schenck FJ. Fast and easy multiresidue method employing acetonitrile extraction/partitioning and "dispersive solid phase extraction" for the determination pesticide residues in produce. J AOAC Int. 2003;86:412.

Barganska Z, Slebioda M, Namiensnik J. Pesticide residues levels in honey from apiaries located in Northern Poland. Food Control. 2013;31:196.

Blasco C, Fernandez M, Pena A, Lino C, Silveira MI, Font G, Pico Y. Assessment of pesticide residues in honey samples from Portugal and Spain. Agric Food Chem. 2003;51:8132-8.

Blasco C, Vazquez-Roig P, Onghena M, Masia A, Pico Y. Analysis of insecticides in honey by liquid chromatogrpahy-ion trap mass spectrometry: comparison of different extraction procedures. J Chromatogr A. 2011;1218:4892-901.

Bogdanov S. Contaminants of bee products. Apidologie. 2006;37:1-18.

Bogdanov S, Jurendic T, Sieber R, Gallmann P. Honey for nutrition and health. J Am Coll Nutr. 2008;27:677-89.

Brodschneider R, Crailsheim K. Nutrition and health in honey bees. Apidologie. 2010;41:278-94

Campillo N, Peñalver R, Aguinaga N, Herna'ndez-Co'rdoba M. Solid- phase microextraction and gas chromatography with atomic emission detection for multiresidue determination of pesticides in honey. Anal Chim Acta. 2006;562:9-15.

Choudhary A, Sharma DC. Pesticide residues in honey samples from Himachal Pradesh (India). Bull Environ Contam Toxicol. 2008:80:417-22.

De Pinho GP, Neves AA, de Queiroz MELR, Silverio FO. Optimization of the liquid-liquid extraction method at low temperature purification (LLE-LTP) for pesticide residue analysis in honey samples by gas-chromatography. Food Control. 2010;21:1307-11

Eissa F, El-Sawi S, Zidan NE. Determining pesticide residues in honey and their potential risk to consumers. Pol J Environ Stud. 2014;23:1573-80.

Erdog rul O". Levels of selected pesticides in honey samples from Kahramanmaras,Turkey. Food Control. 2007;18:866-71.

Fernández MA, Simal LJ. Simplified method for the determination of organochlorine pesticides in honey. Analyst. 1991;116:269-71.

Ferrer C, Lozano A, Agüera A, Girón AJ, Fernández-Alba AR. Overcoming matrix effects using the dilution approach in multiresidue methods for fruits and vegetables. J Chromatogr A. 2011;1218:7634.

Fontana AR, Camargo AB, Altamirano JC. Coacervative microextraction ultrasound-assisted back-extraction technique for determination of organophosphates pesticides in honey samples by gas chromatographymass spectrometry. J Chromatogr A. 2010;1217:6334-41.

Garcia-Chao M, Agruna MJ, Calvete GF, Sakkas V, Llompart M, Dagnac T. Validation of an off line solid phase extraction liquid chromatographytandem mass spectrometry method for the determination of systemic insecticide residues in honey and pollen samples collected in apiaries from NW Spain. Anal Chim Acta. 2010:672:107-33.

Gill RJ, Ramos-Rodriguez O, Raine NE. Combined pesticide exposure severely affects individual- and colony-level traits in bees. Nature. 2012;491:105-8.

Herrera A, Perez AC, Conchello P, Bayarri S, Lazaro R, Yague C, Arino A. Determination of pesticides and PCBs in honey by solid-phase extraction cleanup followed by gas chromatogram with electron-capture and nitrogen-phosphorus detection. Anal Bioanal Chem. 2005;381:695.

Irani M. Determination of pesticides residues in honey samples. Bull Environ Contam Toxicol. 2009;83:818-21

Kittlaus S, Schimanke J, Kempe G, Speer K. Assessment of sample cleanup and matrix effects in the pesticide residue analysis of foods using postcolumn infusion in liquid chromatography-tandem mass spectrometry. J Chromatogr A. 2011;1218:8399.
Kruve A, Künnapas A, Herodes K, Leito I. Matrix effects in pesticide multi-residue analysis by liquid chromatography-mass spectrometry. J Chromatogr A. 2008; 1187:58.

Kujawski MW, Namiesnik J. Challenges in preparing honey samples for chromatographic determination of contaminants and trace residues. TrAC Trends Anal Chem. 2008;27:785-93.

Kujawski MW, Namiesnik J. Levels of 13 multi-class pesticide residues in Polish honeys determined by LC-ESI-MS/MS. Food Control. 2011;22:914-9.

Kujawski MW, Barganska Z, Marciniak K, Miedzianowska E, Kujawski JK, Slebioda M, Namiesnik J. Determining pesticide contamination in honey by LC-ESIMS/MS - comparison of pesticide recoveries of two liquid-liquid extraction based approaches. LWT Food Sci Technol. 2014;56:517-23.

Martel AC, Zeggane S, Auri eres C, Drajnudel P, Faucon JP, Aubert M. Acaricide residues in honey and wax after treatment of honey bee colonies with Apivar or Asuntol50. Apidologie. 2007;38:534-44.

Muli E, Patch H, Frazier M, Frazier J, Torto B, Baumgarten T, Kilonzo J, Kimani JN, Mumoki F, Masiga D, Tumlinson J, Grozinger C. Evaluation of the distribution and impacts of parasites, pathogens, and pesticides on honey bee (Apis mellifera) populations in East Africa. PLoS One. 2014;9(4):e94459.

Mullin CA, Frazier M, Frazier JL, Ashcraft S, Simonds R, Vanengelsdorp D, Pettis JS. High levels of miticides and agrochemicals in North American apiaries: implications for honey bee health. PLoS One. 2010;5(3):e9754.

R Core Team. R: a language and environment for statistical computing. Vienna: $\mathrm{R}$ Foundation for Statistical Computing; 2014. URL http://www.R-project.org/.

Regulation (EC) No 396/2005 with annexes. European Commission: Food Safety/ Plants/Pesticides/Maximum Residue Levels. http://ec.europa.eu/food/plant/ protection/pesticides/community_legislation_en.htm. Accessed 9 July 2015.

Rissato SR, Galhiane MS, De Almeida MV, Gerenutti M, Apon BM. Multiresidue determination of pesticides in honey samples by gas chromatography-mass spectrometry and application in environmental contamination. Food Chem. 2007;101:1719-26.

SANCO/12571/2013. Guidance document on analytical quality control and validation procedures for pesticide residues analysis in food and feed. 2013. Available online: http://ec.europa.eu/food/plant/plant_protection_products/ guidance documents/docs/qualcontrol_en.pdf. Accessed 14 Apr 2015.

Vanengelsdorp D, Meixner MD. A historical review of managed honey bee populations in Europe and the United States and the factors that may affect them. J Invertebr Pathol. 2010;103:S80-95.

Wang J, Kliks MM, Jun S, Li QX. Residues of organochlorine pesticides in honeys from different geographical regions. Food Res Int. 2010;43:2329-34.

Weist L, Bulete A, Giroud B, Fratta C, Amic S, Lambert O, Pouliquen H, Arnaudguilhem C. Multiresidue analysis of 80 environmental contaminants in honeys, honeybees and pollens by one extraction procedure followed by liquid and gas chromatography coupled with mass spectrometric detection. J Chromatogr A. 2011;1218:5743.

\section{Submit your manuscript to a SpringerOpen ${ }^{\circ}$ journal and benefit from:}

- Convenient online submission

- Rigorous peer review

- Immediate publication on acceptance

- Open access: articles freely available online

- High visibility within the field

- Retaining the copyright to your article

Submit your next manuscript at $>$ springeropen.com 